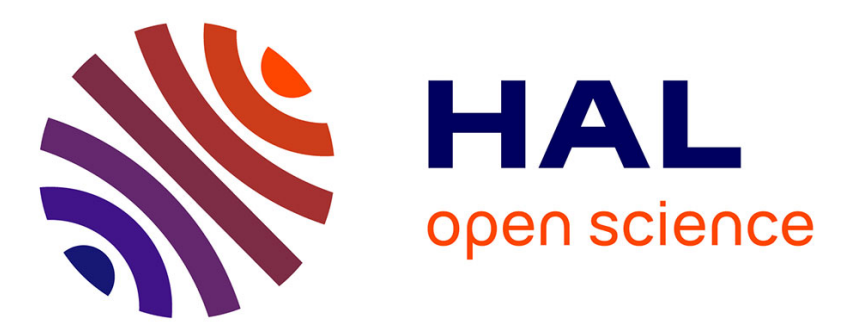

\title{
A combined momentum-interpolation and advection upstream splitting pressure-correction algorithm for simulation of convective and acoustic transport at all levels of Mach number \\ Yann Moguen, Pascal Bruel, Erik Dick
}

\section{To cite this version:}

Yann Moguen, Pascal Bruel, Erik Dick. A combined momentum-interpolation and advection upstream splitting pressure-correction algorithm for simulation of convective and acoustic transport at all levels of Mach number. Journal of Computational Physics, 2019, 384, pp.16-41. 10.1016/j.jcp.2019.01.029 . hal-02064848v2

\author{
HAL Id: hal-02064848 \\ https://hal.inria.fr/hal-02064848v2
}

Submitted on 22 Oct 2019

HAL is a multi-disciplinary open access archive for the deposit and dissemination of scientific research documents, whether they are published or not. The documents may come from teaching and research institutions in France or abroad, or from public or private research centers.
L'archive ouverte pluridisciplinaire HAL, est destinée au dépôt et à la diffusion de documents scientifiques de niveau recherche, publiés ou non, émanant des établissements d'enseignement et de recherche français ou étrangers, des laboratoires publics ou privés. 


\title{
A combined momentum-interpolation and advection upstream splitting pressure-correction algorithm for simulation of convective and acoustic transport at all levels of Mach number
}

\author{
Yann Moguen ${ }^{\mathrm{a}, *}$, Pascal Bruel ${ }^{\mathrm{b}}$ and Erik Dick ${ }^{\mathrm{c}}$ \\ ${ }^{a}$ Univ. Pau \& Pays Adour, SIAME/LMAP, 64013 Pau, France \\ ${ }^{\mathrm{b}}$ CNRS/Univ. Pau \& Pays Adour, LMAP/Inria Cagire Team, 64013 Pau, France \\ ${ }^{\mathrm{c}}$ Ghent University, Department of Flow, Heat and Combustion Mechanics, \\ Sint-Pietersnieuwstraat, 41, 9000 Gent, Belgium
}

\begin{abstract}
A pressure-correction algorithm is presented for compressible fluid flow regimes. It is well-suited to simulate flows at all levels of Mach number with smooth and discontinuous flow field changes, by providing a precise representation of convective transport and acoustic propagation. The co-located finite volume space discretization is used with the AUSM flux splitting. It is demonstrated that two ingredients are essential for obtaining good quality solutions: the presence of an inertia term in the face velocity expression; a velocity difference diffusive term in the face pressure expression, with a correct Mach number scaling to recover the hydrodynamic and acoustic low Mach number limits. To meet these two requirements, a new flux scheme, named MIAU, for Momentum Interpolation with Advection Upstream splitting is proposed.
\end{abstract}

Key words: All-Mach number scheme ; momentum interpolation ; advection upstream splitting ; co-located finite volume ; convective transport ; acoustic propagation

This work is dedicated to the memory of our friend and colleague Jan Vierendeels.

\section{Introduction}

Distinguishing between density-based and pressure-based methodologies in computational fluid dynamics (CFD) is a common way of categorizing the various approaches

\footnotetext{
* Corresponding author. Tel.: +33 559407552.

Email address: yann. moguen@univ-pau. fr(Yann Moguen).
} 
of solving the governing equations of fluid motion. Choosing one approach or the other is clearly driven by the type of flow as well as by the background, or even the preference, of the developer. Among the underlying arguments behind that choice, the versatility of the method, its robustness, its simplicity of implementation and its efficiency (convergence properties, parallelization in a high performance computing environment) are certainly the features that do matter most when elaborating one's choice. A comprehensive review on methods for CFD can be found in the textbooks by LeVeque [1], Toro [2], Tucker [3] or Wesseling [4], to name a few. Concerning specifically pressure-based methods, there exists a large body of literature which is not referenced here, but the interested reader may supplement the information given in the aforementioned textbooks by the ten-year old review by Acharya et al. [5]. Whenever elaborating a pressure-based algorithm within a co-located finite volume framework, two crucial aspects have to be carefully addressed: (i) The equation for derivation of the pressure correction has to be properly selected; (ii) the expressions of the velocity and the pressure at the face between two adjacent cells have to be established. Regarding point $(i)$, a significant number of proposals rely on the continuity equation to derive the pressure correction (SIMPLE, PISO) or the pressure (SIMPLER) equation, to single out the most popular approaches. Historically, the reason is that such methods were primarily intended for the incompressible flow regime. But for the solution of the full compressible flow equations at low Mach number, it was demonstrated by Klein [6] that a priori, the algorithm development should be based on attributing to the continuity equation the role of advecting the entropy, while the velocity field divergence constraint derives naturally from the energy equation. In our earlier proposals for low [7] or all [8] Mach number flow simulations on which the present contribution is based, Klein's viewpoint was adopted, i.e. the pressure correction equation was derived from the energy equation. Concerning point (ii) for compressible flows, there exist broadly speaking two categories of methods. One is based on solving approximately a Riemann problem at each face using characteristic equations derived thanks to the hyperbolic nature of the system of the compressible flow equations. Approximate Riemann solvers are principally aimed at accurately representing shocks, contact discontinuities or expansion fans in high Mach number flows. For smooth solutions of low Mach number flows involving acoustic wave propagation, they are intrinsically very dissipative and call for specific adaptations such as the inclusion of an inertia term in the face velocity expression [8]. The second category, referred to as momentum or pressure weighted interpolation, is based on a preliminary discretization of the momentum equations on a dual mesh to derive the face velocity, possibly combined with a flux splitting based expression of the face pressure. The methodology was initially meant for smooth flow solutions and first proposed by Rhie and Chow [9]. Many variants were subsequently developed for steady flows $[10,11]$ as well as for unsteady low Mach number flows featuring travelling acoustic waves $[7,12,13]$. For handling acoustic propagation, the key ingredient proved to be the introduction of an inertia term in the definition of the face velocity [7], a term which is absent in basic versions of approximate Riemann solvers.

Although no dramatic breakthrough has been recorded during the last twenty years, continuous improvements have been obtained in order to enhance either the basic capabilities or the versatility of pressure-based methods. Regarding the latter, the capability of solving 
the full set of compressible Euler or Navier-Stokes equations over an as wide as possible range of Mach number, possibly involving a complex equation of state, while preserving the correctness of the asymptotic behavior when the Mach number tends to zero, has been the subject of a significant number of recent studies (see e.g. [14,15]). In that framework, contributing to the improvement of a pressure-based approach for solving the full compressible flow equations within a finite volume co-located framework has been an objective we pursued during the last ten years. With this motivation, we studied combinations of Riemann solver based algorithms and momentum interpolation methods. In [7], we presented an algorithm with finite-volume second-order TVD space discretization with co-located variables and AUSM flux-vector splitting. The time discretization was second-order backward differencing. The face velocity for all equations was derived from a time-consistent momentum interpolation of Rhie-Chow type applied to preliminary discretized momentum and continuity equations, with the face velocity and face pressure by the low Mach number formulation of $\mathrm{AUSM}^{+}$, called $\mathrm{AUSM}^{+}$-up, but without velocity and pressure diffusion terms. In the final discretization, the momentum-interpolated face velocity was used in all equations, together with the previous expression for face pressure. The solution was obtained by pressure-correction derived from the energy equation. This time-consistent momentum interpolation algorithm is suitable for steady and unsteady low Mach number flows with smooth variation of flow parameters. Good performance was demonstrated for propagation of acoustic waves in low Mach number flows. Not illustrated in [7] was that this algorithm does not function well for Riemann problems. In [16], a time-step dependent modification of the coefficient of the pressure diffusion term in the $\mathrm{AUSM}^{+}$-up face velocity expression was derived, based on the Mach number scaling of the time-consistent momentum interpolation method. The face pressure was the $\mathrm{AUSM}^{+}$-up expression without the velocity diffusion term. Compared to the $\mathrm{AUSM}^{+}$-up scheme, the performance for propagation of acoustic waves in low Mach number flows is much improved, but it does not reach fully that of the time-consistent momentum interpolation method. In [8], an inertia term was added to the face velocity expression of the $\mathrm{AUSM}^{+}$-up scheme, similar to the inertia term in the time-consistent momentum interpolation method. This face velocity was combined with the $\mathrm{AUSM}^{+}$-up face pressure without velocity diffusion term. The obtained scheme, denoted by AUSM-IT, has the same low Mach number scaling as the time-consistent momentum interpolation method and it functions equally well for propagation of acoustic waves in low Mach number flows. The scheme functions also well for low Mach number Riemann problems. For such problems, the $\mathrm{AUSM}^{+}$-up scheme functions also well, while the $\mathrm{AUSM}^{+}$scheme and the momentum interpolation method of [7] produce oscillations around discontinuities. For high Mach number Riemann problems, the proposed scheme produces sometimes a slight sonic glitch in expansion fans and oscillations around shocks. The same deficiencies were observed with the $\mathrm{AUSM}^{+}$-up scheme. In [17], it was demonstrated that oscillations in low Mach number Riemann problems by the time-consistent momentum interpolation method are reduced by replacing the interpolation of the momentum parts, associated to the adjacent nodes of a face, by interpolation of the momenta themselves. This makes the method similar to the $\mathrm{AUSM}^{+}$-up scheme with an added inertia term. The scheme functions also well for propagation of acoustic waves in low Mach number flows, but not for high Mach number Riemann problems. So, none of the $\mathrm{AUSM}^{+}, \mathrm{AUSM}^{+}$-up, AUSM-IT 
schemes and the time-consistent momentum interpolation methods of [7] and [17] function equally well for low and high Mach number flows and for flows with smooth and discontinuous flow parameter changes.

From an application viewpoint, many systems of practical interest such as jet engine combustion chambers or turbomachinery components of gas turbines are featuring (simultaneously or not) a large diversity of flow regimes and structures, that call for the development of versatile simulations tools. With such a general context in mind, the present contribution describes a pressure-based algorithm that permits an accurate representation of discontinuities, smooth expansion fans and smooth acoustic waves over a range of flow Mach number from 0 to 239 in the presented numerical tests. The proposed predictorcorrector algorithm inherits its general structure from our previous method [7] for low Mach number flows, which is modified in order to incorporate the following improvements:

(1) A simplified time-consistent momentum interpolation that avoids the pre-estimation step of [7], i.e. the double density and momentum stepping, while maintaining the high quality of the results but simplifying the flowchart of the algorithm. The basic discretization is done with the $\mathrm{AUSM}^{+}$method, because of its good properties for solving steady flows and Riemann problems at high Mach number.

(2) A new face pressure formulation.

The combination of the momentum interpolation method for the face velocity expression with the proposed face pressure expression has the beneficial effect of stabilizing low Mach number flow simulations, i.e. preventing the odd-even decoupling, while permitting an accurate representation of Riemann problems, both in low and high Mach number flows. This specific combination is called here the Momentum Interpolation with Advection Upstream splitting, or MIAU, scheme.

The layout of the paper is the following: In section 2, the low Mach number behavior of the continuous system of governing equations is briefly described. Conclusions are drawn on the possible formulation of expressions of the face velocity and the face pressure. In section 3, the retained expressions of the face pressure and velocity are presented as well as the pressure correction algorithm. In section 4, the proposed algorithm is compared to other momentum-interpolation methods. Sections 5 to 9 are presentations of the results of various tests carried out to evidence the quality and the versatility of the proposed methodology. 


\section{The continuous system and its asymptotic behavior}

\subsection{The continuous system}

We take as fluid an ideal and perfect gas. The basic primitive flow variables are pressure $p$, density $\varrho$ and velocity $\boldsymbol{v}$. The conservative form of the Euler equations reads as

$$
\begin{aligned}
& \partial_{t} \varrho+\nabla \cdot(\varrho \boldsymbol{v})=0, \\
& \partial_{t}(\varrho \boldsymbol{v})+\nabla \cdot(\varrho \boldsymbol{v} \otimes \boldsymbol{v})+\nabla p=0, \\
& \partial_{t}(\varrho E)+\nabla \cdot(\varrho H \boldsymbol{v})=0,
\end{aligned}
$$

where $t, E$ and $H$ represent time, specific total energy and specific total enthalpy. Relationships are

$$
\varrho H=\varrho E+p, \quad E=e+\frac{1}{2}\|\boldsymbol{v}\|^{2}, \quad H=h+\frac{1}{2}\|\boldsymbol{v}\|^{2}, \quad h=e+\frac{p}{\varrho},
$$

where $e$ is the specific internal energy and $h$ the specific enthalpy. A relation between $e$ and the basic variables $p$ and $\varrho$ is given by an equation of state, $e=e(p, \varrho)$. For an ideal and perfect gas, it is

$$
e=\frac{p}{\varrho(\gamma-1)}
$$

with $\gamma$ the ratio of specific heats.

For later use, the one-dimensional equations are also considered, which may be written as

$$
\partial_{t} Q+\partial_{x} F(Q)=0
$$

with

$$
Q=\left[\begin{array}{c}
\varrho \\
\varrho v \\
\varrho E
\end{array}\right], \quad F(Q)=\left[\begin{array}{c}
\varrho v \\
\varrho v v+p \\
\varrho H v
\end{array}\right],
$$

where $x$ represents the space coordinate.

\subsection{Advection and pressure parts}

In the present work, the numerical scheme uses the concept of flux splitting, which means that the flux balance part of the conservative equations (1)-(3) is split into an advection part and a pressure part. We follow the splitting of the AUSM [18,19], which considers the 
terms under the divergence operator as belonging to the advection part, and the gradient of the pressure as belonging to the pressure part. For the one-dimensional system (4), this means

$$
F^{\mathrm{A}}(Q)=\left[\begin{array}{c}
\varrho \\
\varrho v \\
\varrho H
\end{array}\right] v \quad, \quad F^{\mathrm{P}}(Q)=\left[\begin{array}{c}
0 \\
p \\
0
\end{array}\right] .
$$

A co-located finite volume space discretization is considered, with the primitive variables and derived quantities stored at cell centers. The advection is discretized in an upwind way, detailed later. The discretization requires the definition of face values of velocity and pressure. For correct low Mach number behavior, these face quantities have to obey some conditions derived hereafter.

It should be mentioned that the splitting of the flux vector into advection and pressure parts is not unique. Several possibilities exist such as the one proposed by Yabe and Wang [20], adopted by Zha and Bilgen [21], or that by Toro and Vázquez-Cendón [22]. For an analysis of the differences between the possible choices, the interested reader is referred to [22]. The method presented here is built for the AUSM splitting and uses the AUSM ${ }^{+}$ definition of the face velocity in the basic discretization. Formally, the method can be used for the other splittings too, but it does not always lead to a good quality solution, or it does not allow a solution at all. For another splitting than AUSM, the face velocity and the face pressure must be adapted.

\subsection{Non-dimensionalisation}

Reference values for pressure $p_{\mathrm{r}}$, density $\varrho_{\mathrm{r}}$ and velocity $v_{\mathrm{r}}$ (thought of as advective) are used. A reference Mach number is then defined as $\mathrm{M}_{\mathrm{r}}=v_{\mathrm{r}} / \sqrt{p_{\mathrm{r}} / \varrho_{\mathrm{r}}}$. Reference values for duration $t_{\mathrm{r}}$ (thought of as advective) and length $l_{\mathrm{r}}$ (to be chosen hereafter) are also introduced. A reference Strouhal number is then defined as $\mathrm{St}_{\mathrm{r}}=\left(l_{\mathrm{r}} / v_{\mathrm{r}}\right) / t_{\mathrm{r}}$. The dimensionless Euler equations are

$$
\begin{aligned}
& \mathrm{St}_{\mathrm{r}} \partial_{t} \varrho+\nabla \cdot(\varrho \boldsymbol{v})=0, \\
& \mathrm{St}_{\mathrm{r}} \partial_{t}(\varrho \boldsymbol{v})+\nabla \cdot(\varrho \boldsymbol{v} \otimes \boldsymbol{v})+\frac{1}{\mathrm{M}_{\mathrm{r}}^{2}} \nabla p=0, \\
& \mathrm{St}_{\mathrm{r}} \partial_{t}(\varrho E)+\nabla \cdot(\varrho H \boldsymbol{v})=0,
\end{aligned}
$$

with

$$
\varrho H=\varrho E+p, \quad E=e+\frac{1}{2} \mathrm{M}_{\mathrm{r}}^{2}\|\boldsymbol{v}\|^{2}, \quad H=h+\frac{1}{2} \mathrm{M}_{\mathrm{r}}^{2}\|\boldsymbol{v}\|^{2}, \quad h=e+\frac{p}{\varrho}, e=\frac{p}{\varrho(\gamma-1)} .
$$




\subsection{The hydrodynamic low Mach number limit}

For ease of presentation and clarity, periodic boundary conditions are assumed and the spatial domain considered is the torus defined by

$$
\mathbb{T}=[\mathbb{R} /(L \mathbb{Z})]^{d} \text { with } L \gg l_{\mathrm{r}}
$$

where $d=1,2$ or 3 .

For this limit, the reference length $l_{\mathrm{r}}$ is chosen as an advective quantity, i.e. $l_{\mathrm{r}}=\mathcal{O}\left(t_{\mathrm{r}} v_{\mathrm{r}}\right)$. This corresponds to $\mathrm{St}_{\mathrm{r}}=\mathcal{O}(1)$. The toric domain is then denoted by $\mathbb{T}_{\text {advect }}$. It is then assumed that the pressure can be expanded in the low Mach number limit as

$$
p\left(\boldsymbol{x}, t, \mathrm{M}_{\mathrm{r}}\right)=\sum_{n=0}^{N} \mathrm{M}_{\mathrm{r}}^{n} p^{(n)}(\boldsymbol{x}, t)+o\left(\mathrm{M}^{N}\right), N=0,1,2,
$$

and that similar expansions hold for all other quantities. The substitution of these expansions into the dimensionless Euler equations, with periodic boundary conditions, gives on $\mathbb{T}_{\text {advect }}[23-25]$

$$
\begin{aligned}
& \partial_{t} \varrho^{(0)}+\boldsymbol{v}^{(0)} \cdot \nabla \varrho^{(0)}=0 \\
& \varrho^{(0)} \partial_{t} \boldsymbol{v}^{(0)}+\varrho^{(0)} \boldsymbol{v}^{(0)} \cdot \nabla \boldsymbol{v}^{(0)}+\nabla p^{(2)}=0 \\
& \mathrm{~d}_{t} p^{(0)}=0 \\
& \nabla \cdot \boldsymbol{v}^{(0)}=0 .
\end{aligned}
$$

The involved pressure terms are the thermodynamic pressure $p^{(0)}$ and the hydrodynamic pressure $p^{(2)}$. The thermodynamic pressure is constant in the flow field. The hydrodynamic pressure is the part of the pressure that scales quadratically with the Mach number. The part that scales linearly with the Mach number, $p^{(1)}$, is the acoustic pressure. In the hydrodynamic low Mach number limit of the Euler equations, which means looking at propagation on the (small) advective length scale, acoustic fluctuations are not relevant because their characteristic wavelength is much larger than the chosen length scale.

Under the assumption of uniform $\varrho^{(0)}$, the hydrodynamic kinetic energy on the torus $\mathbb{T}_{\text {advect }}$ may be defined as

$$
E_{\text {kin }}=\int_{\mathbb{T}_{\text {advect }}} \frac{1}{2} \varrho^{(0)}\left\|\boldsymbol{v}^{(0)}\right\|^{2}
$$

With Eqs. (7)-(9), it satisfies

$$
\mathrm{d}_{t} E_{\mathrm{kin}}=0
$$




\subsection{The acoustic low Mach number limit}

For this limit, the reference length $l_{\mathrm{r}}$ is chosen as an acoustic quantity, i.e. $l_{\mathrm{r}}=\mathcal{O}\left(t_{\mathrm{r}} \sqrt{p_{\mathrm{r}} / \varrho_{\mathrm{r}}}\right)$. This corresponds to $\mathrm{St}_{\mathrm{r}}=\mathcal{O}\left(1 / \mathrm{M}_{\mathrm{r}}\right)$. The toric domain, defined similarly as before, is now denoted by $\mathbb{T}_{\text {acoust }}$. A variable relevant to observe acoustic scale fluctuations is introduced as

$$
\boldsymbol{\xi}=\mathrm{M}_{\mathrm{r}} \boldsymbol{x} .
$$

We assume that the pressure can be expanded in the low Mach number limit as

$$
p\left(\boldsymbol{x}, t, \mathrm{M}_{\mathrm{r}}\right)=\sum_{n=0}^{N} \mathrm{M}_{\mathrm{r}}^{n} p^{(n)}(\boldsymbol{x}, \boldsymbol{\xi}, t)+o\left(\mathrm{M}^{N}\right), N=0,1,2,
$$

and that similar expansions hold for all other quantities. From the dimensionless Euler

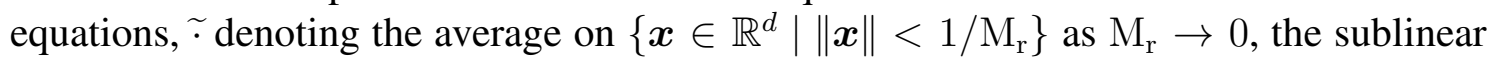
growth condition gives $[6,25]$

$$
\begin{aligned}
& \widetilde{\varrho^{(0)}} \partial_{t} \widetilde{\boldsymbol{v}^{(0)}}+\nabla_{\boldsymbol{\xi}} p^{(1)}=0, \\
& \partial_{t} p^{(1)}+\gamma p^{(0)} \nabla_{\boldsymbol{\xi}} \cdot \widetilde{\boldsymbol{v}^{(0)}}=0 .
\end{aligned}
$$

From the previous equations, one may derive the acoustic wave equation:

$$
\partial_{t t} p^{(1)}-\nabla_{\boldsymbol{\xi}} \cdot\left(c_{0}^{2} \nabla_{\boldsymbol{\xi}} p^{(1)}\right)=0
$$

where $c_{0}=c_{0}(\boldsymbol{\xi}, t)=\sqrt{\gamma p^{(0)}(t) / \widetilde{\varrho^{(0)}}(\boldsymbol{\xi})}$. Thus, $p^{(1)}$ and $\widetilde{\boldsymbol{v}^{(0)}}$ are identified as acoustic quantities. In the acoustic low Mach number limit of the Euler equations, which means looking at propagation on the (large) acoustic length scale, advective propagation is not relevant because it happens on a small length scale.

Under the assumption of constant density $\widetilde{\varrho^{(0)}}$, the acoustic energy on the torus $\mathbb{T}_{\text {acoust }}$ may be defined as

$$
E_{\text {acoust }}=\int_{\mathbb{T}_{\text {acoust }}}\left[\frac{1}{2} \widetilde{\varrho^{(0)}}\left\|\widetilde{\boldsymbol{v}^{(0)}}\right\|^{2}+\frac{1}{2} \frac{\left(p^{(1)}\right)^{2}}{\varrho^{(0)}\left(c^{(0)}\right)^{2}}\right] .
$$

With Eqs. (10)-(11), it satisfies:

$$
\mathrm{d}_{t} E_{\text {acoust }}=0 .
$$

\subsection{Conditions on face values}

With a co-located finite volume discretization, the limit equations (7)-(9) and (10)-(11) are satisfied at the level of the cells due to the conservative way of discretization. For 
good low Mach number behavior of the discretized equations, face values of velocity and pressure have to be chosen in accordance with the limit equations. For obeying the acoustic low Mach number limit, the density and the velocity of sound at a face may be obtained by interpolation, and the face pressure and face velocity derived from the characteristic equations associated to the set (10)-(11). For the linearized one-dimensional Euler equations, the characteristic equations are

$$
\mathrm{d} p \pm \varrho c \mathrm{~d} v=0 \quad \text { along } \quad \mathrm{d}_{t} x=v \pm c .
$$

Pressure and velocity at a face can be taken at the state in the wedge region between the wave paths associated to $v \pm c$, usually called the star region [2]. By indicating cell centers with the subscript $i$ and cell faces with the subscript $i+1 / 2$, we may write for a $(v+c)$-wave

$$
p_{i+1 / 2}-p_{\mathrm{L}}+\varrho_{i+1 / 2} c_{i+1 / 2}\left(v_{i+1 / 2}-v_{\mathrm{L}}\right)=0,
$$

and for a $(v-c)$-wave

$$
p_{i+1 / 2}-p_{\mathrm{R}}-\varrho_{i+1 / 2} c_{i+1 / 2}\left(v_{i+1 / 2}-v_{\mathrm{R}}\right)=0,
$$

where $\mathrm{L}$ and $\mathrm{R}$ denote the left and right hand states of the Riemann problem. With a discrete field representation, these states are typically defined by slope-limited extrapolation of cell-center values.

Combination of equations (13) and (14) results in

$$
v_{i+1 / 2}=\frac{1}{2}\left(v_{\mathrm{L}}+v_{\mathrm{R}}\right)+\frac{1}{2} \frac{p_{\mathrm{L}}-p_{\mathrm{R}}}{\varrho_{i+1 / 2} c_{i+1 / 2}},
$$

and

$$
p_{i+1 / 2}=\frac{1}{2}\left(p_{\mathrm{L}}+p_{\mathrm{R}}\right)+\frac{1}{2} \varrho_{i+1 / 2} c_{i+1 / 2}\left(v_{\mathrm{L}}-v_{\mathrm{R}}\right) .
$$

The equations (15) and (16) can be used for simulation of propagation of acoustic waves in a low Mach number flow, but they do not comply with the limit equations (7)-(9) for hydrodynamic transport in a low Mach number flow.

The momentum equations (8) and (10) are common to the two limiting cases. So, we may replace the characteristic expression for face velocity (15) by the face velocity from a momentum equation obtained by interpolation of the momentum equations in the cells. We will discuss the practical aspects of such an interpolation in a later section. But, we should already stress that for a good representation of acoustic propagation in a low Mach number flow, the inertia term and the pressure gradient term in (10) have to be expressed locally at a face. The same is then automatically necessary for the inertia and pressure gradient terms in the interpolated momentum equation. This means that the interpolation can only be done for the momentum flux balance terms. 
Expression (16) for pressure obeys the $p^{(1)}$ character of the pressure, imposed by the momentum equation (10), which means that pressure perturbations scale linearly with a varying Mach number. This is not in accordance with the required $p^{(2)}$ character of the pressure in the hydrodynamic limit by the momentum equation (8), which requires that pressure perturbations scale with the square of the Mach number. The $p^{(2)}$ scaling can be obtained by multiplication of the coefficient of the velocity difference term in (16) by a function $f(M)$ which becomes linear in the Mach number for small values of Mach number and becomes unity for high Mach number values. This means replacing (16) by

$$
p_{i+1 / 2}=\frac{1}{2}\left(p_{\mathrm{L}}+p_{\mathrm{R}}\right)+\frac{1}{2} f(M) \varrho_{i+1 / 2} c_{i+1 / 2}\left(v_{\mathrm{L}}-v_{\mathrm{R}}\right) .
$$

Such a modification is quite common in adaptations of face values for pressure obtained by a discretization method suitable for high Mach number flows. We will discuss some examples and make our own choice of the multiplication function in a later section.

The expression (17) is still good for simulation of acoustic propagation of continuous perturbations in low Mach number flows, because velocity differences become then small and pressure is then continuous. So, the limit behavior required by the equations (10)-(11) is not violated at the discrete level, although the scaling of the pressure becomes a $p^{(2)}$ scaling. We will, of course, verify that this is true by numerical tests.

There is no a priori guarantee that an expression of a face velocity obtained by interpolation of momentum equations combined with an expression of face pressure by a modified characteristic relation may function well for Riemann problems. For good representation of Riemann problems by a discrete method, it is required that the discretization is done with the equations in conservative form and that the conservation laws are expressed on the cells in a consistent way. For an AUSM-type method, this means that the transporting velocity in the flux terms has to be the same in all equations. A plausible condition for avoiding oscillations around discontinuities is then that density, velocity and pressure at the cell faces have to be within the intervals spanned by the values in the neighboring cell centers. In principle, by the momentum interpolation and by the expression of the face pressure in which the coefficient of the velocity difference term is reduced, this is guaranteed. Again, we will verify by tests that the expressions that we finally choose function well for Riemann problems.

\section{The pressure-correction scheme and discretization in a one-dimensional space}

The pressure-correction process is detailed for the calculation of the flow variables at the cell $i$ with $1 \leq i \leq N$. Each time-step $n \rightarrow n+1$ is decomposed into predictioncorrection $k$-indexed iterations (for the first iteration, the $k=1$ values are taken equal to the $n$ values). The superscripts * and ' denote the predicted quantities and correction values of each iteration $k$, respectively. For simplicity, the discretizations are written for a onedimensional flow configuration with constant values of time step and cell size. Extension 
of the discretization to non-constant values is immediate. The face indexed by $i+1 / 2$ is selected to expose the various aspects related to the determination of the transporting velocity or the pressure at a face. The subscripts $L$ and $R$ designate the left and right states at a face $i+1 / 2$, obtained by extrapolation with a symmetric slope limiter $\psi$, as

$$
\phi_{\mathrm{L}}=\phi_{i}+\frac{1}{2} \psi_{i}(\phi), \phi_{\mathrm{R}}=\phi_{i+1}-\frac{1}{2} \psi_{i+1}(\phi)
$$

where the Minmod slope limiter is used, namely

$$
\psi_{i}(\phi)=\operatorname{Minmod}\left(\phi_{i}-\phi_{i-1}, \phi_{i+1}-\phi_{i}\right)
$$

with

$$
\operatorname{Minmod}(a, b)=\frac{1}{2}[\operatorname{sign}(a)+\operatorname{sign}(b)] \min \{|a|,|b|\} .
$$

To simplify the presentation, the velocity is assumed to be positive. This means that, for a convected quantity $\phi$, the left value $\phi_{\mathrm{L}}$ is chosen. We first recall definitions that will provide the necessary ingredients when introducing the MIAU scheme in section 3.4.

\subsection{The AUSM+ face velocity and face pressure}

The face velocity of an AUSM family scheme is based on a common velocity of sound at the face $c_{i+1 / 2}$ and an interpolated Mach number $M_{i+1 / 2}$, with, for $\mathrm{AUSM}^{+}$[18],

$$
\begin{gathered}
v_{i+1 / 2}^{\mathrm{AUSM}^{+}}=c_{i+1 / 2} M_{i+1 / 2}, \quad c_{i+1 / 2}=\min \left\{\tilde{c}_{\mathrm{L}}, \tilde{c}_{\mathrm{R}}\right\} \\
\tilde{c}_{\mathrm{L}}=\left(c_{\mathrm{L}}^{*}\right)^{2} / \max \left\{c_{\mathrm{L}}^{*},\left|v_{\mathrm{L}}\right|\right\}, \tilde{c}_{\mathrm{R}}=\left(c_{\mathrm{R}}^{*}\right)^{2} / \max \left\{c_{\mathrm{R}}^{*},\left|v_{\mathrm{R}}\right|\right\},\left(c_{\mathrm{L}, \mathrm{R}}^{*}\right)^{2}=\frac{2(\gamma-1)}{\gamma+1} H_{\mathrm{L}, \mathrm{R}}, \\
M_{i+1 / 2}=f_{M}^{+}\left(M_{\mathrm{L}}\right)+f_{M}^{-}\left(M_{\mathrm{R}}\right), \\
\text { with } f_{M}^{ \pm}(m)= \begin{cases}\frac{1}{2}(m \pm|m|) & ,|m| \geq 1, \\
\pm \frac{1}{4}(m \pm 1)^{2} \pm \frac{1}{8}\left(m^{2}-1\right)^{2} & ,|m|<1\end{cases}
\end{gathered}
$$

$$
M_{\mathrm{L}, \mathrm{R}}=v_{\mathrm{L}, \mathrm{R}} / c_{i+1 / 2},
$$

where $\mathrm{L}$ and $\mathrm{R}$ denote left and right hand face states, obtained by slope-limited extrapolation. For the $\mathrm{AUSM}^{+}$face pressure, the Mach number dependent interpolation formula is

$$
\begin{aligned}
p_{i+1 / 2}^{\mathrm{AUSM}^{+}}= & f_{p}^{+}\left(M_{\mathrm{L}}\right) p_{\mathrm{L}}+f_{p}^{-}\left(M_{\mathrm{R}}\right) p_{\mathrm{R}}, \\
& \text { with } f_{p}^{ \pm}(m)= \begin{cases}\frac{1}{2}(1 \pm \operatorname{sign}(m)) & ,|m| \geq 1, \\
\frac{1}{4}(m \pm 1)^{2}(2 \mp m) \pm \frac{3}{16} m\left(m^{2}-1\right)^{2} & ,|m|<1 .\end{cases}
\end{aligned}
$$


3.2 The face pressure for low-diffusion or low-dissipation formulation in low Mach number flows

For an AUSM family scheme, the expressions for face velocity and face pressure are derived from the characteristic equations associated to the one-dimensional Euler equations (12). For the basic AUSM definitions (19)-(23), only the observation that the characteristic speeds are $v \pm c$ is taken as a motivation for the definition of the polynomials (21) and (23) as functions of $m \pm 1$. Low Mach number adaptations are based on the characteristic relations (15) and (16).

There are two vital aspects of these formulae for use with low Mach number flows. First, the pressure difference term in the velocity expression (15) is necessary for avoiding oddeven decoupling. The second is that the scaling, as a function of the Mach number, of the coefficients of the pressure difference term in (15), and the velocity difference term in (16), is not correct for low values of the Mach number in the hydrodynamic limit. These coefficients should, respectively, scale inversely proportional and directly proportional to the magnitude of the flow velocity instead of the velocity of sound (see section 2.6).

The polynomial interpolation formulae (21) and (23) imply pressure and velocity difference terms, which can be made visible by rewriting the expressions as an average term plus difference terms. Thus, the face pressure (23) may be written as

$$
\begin{aligned}
p_{i+1 / 2}=\frac{1}{2}\left(p_{\mathrm{L}}+p_{\mathrm{R}}\right)+\frac{1}{2}\left[f_{p}^{+}\left(M_{\mathrm{L}}\right)\right. & \left.-f_{p}^{-}\left(M_{\mathrm{R}}\right)\right]\left(p_{\mathrm{L}}-p_{\mathrm{R}}\right) \\
+ & \frac{1}{2} f_{p}\left[f_{p}^{+}\left(M_{\mathrm{L}}\right)+f_{p}^{-}\left(M_{\mathrm{R}}\right)-1\right]\left(p_{\mathrm{L}}+p_{\mathrm{R}}\right),
\end{aligned}
$$

with $f_{p}=1$ for formula (23). The term $f_{p}^{+}\left(M_{\mathrm{L}}\right)+f_{p}^{-}\left(M_{\mathrm{R}}\right)-1$ is proportional to $v_{\mathrm{L}}-v_{\mathrm{R}}$ and the correct scaling is obtained by a factor $f_{p}$ which becomes proportional to the Mach number for low Mach number flow. Such a form of scaling was used by Edwards and Liou [26] in a formulation called Low-Diffusion Flux Splitting Scheme (LDFSS), but with a supplementary scaling factor on the coefficient of the pressure difference term in (24). A simplified formulation was introduced by Shima and Kitamura [27,28] in a scheme called Simple Low-diffusion AUSM (SLAU), with only an $f_{p}$ factor in (24). The $\mathrm{AUSM}^{+}$-up method of Liou [19] is somewhat different in the sense that a velocity difference term is added to the face pressure expression (23). Moreover, a coefficient in the interpolation polynomial is made dependent on the Mach number, with the objective to reach an interpolation close to an average for low Mach number.

The three cited methods can all be seen as manipulations aimed at obtaining a face pressure expression close to the characteristic expression (16), but with the coefficient of the velocity difference term multiplied by a factor which becomes proportional to the Mach number for low Mach number. So, the correct scaling can be obtained by a modification 
of the expression (16) into

$$
p_{i+1 / 2}=\frac{1}{2}\left(p_{\mathrm{L}}+p_{\mathrm{R}}\right)+\frac{1}{2} f_{p} \varrho_{i+1 / 2} c_{i+1 / 2}\left(v_{\mathrm{L}}-v_{\mathrm{R}}\right) .
$$

In this expression, we use the factor $f_{p}$ of Shima and Kitamura [27]. This factor, similar to, but simpler than the $f_{a}$ function of the $\mathrm{AUSM}^{+}$-up scheme [19], reads as

$$
f_{p}=2 \widetilde{M}_{i+1 / 2}-\widetilde{M}_{i+1 / 2}^{2}, \quad \widetilde{M}_{i+1 / 2}=\min \left\{1, \sqrt{\frac{v_{\mathrm{L}}^{2}+v_{\mathrm{R}}^{2}}{2 c_{i+1 / 2}^{2}}}\right\} .
$$

With the expression (25), the face pressure stays close to the characteristic expression for low and high Mach number flows. This creates the best chances for good solution of Riemann problems at low and high Mach number.

The necessary modifications to the expression (19) of the face velocity are more involved. We do not use one of these developed by Edwards and Liou [26], Liou [19] or Shima and Kitamura [27,28], since they were only meant for coping with the hydrodynamic low Mach number limit. Instead, we obtain the necessary pressure-velocity coupling and the correct Mach number scaling for both the hydrodynamic and acoustic limits by momentum interpolation, as detailed in a following section.

The change made to the coefficient of the velocity difference term in the expression of the face pressure $(17,25)$ is similar to adaptations done by Rieper [29] and $\mathrm{Li}$ and $\mathrm{Gu}$ [30] to the Roe flux-difference scheme for making it suitable for simulations of flows in the hydrodynamic low Mach number limit. The face flux vector by the Roe scheme is the sum of an average flux and a matrix multiplied with the vector of differences of the conservative variables. The matrix can be decomposed into the product of a right eigenvector matrix, an eigenvalue matrix and a left eigenvector matrix. The eigenvalues are $v+c, v$ and $v-c$ (in one dimension). In the method by Rieper, the product of the left eigenvector matrix and the vector of differences of the conservative variables is written as the product of a matrix and the vector of differences of the primitive variables $(\varrho, v$, $p$, in one dimension). The term in $\Delta v$ causes a $p^{(1)}$ type contribution to the pressure, as already shown by Guillard and Viozat [31]. This contribution is the cause of the erroneous behavior in the hydrodynamic low Mach number limit. The cure proposed by Rieper is to replace the $\Delta v$ term by $f(M) \Delta v$. This change converts the contribution of the term into $p^{(2)}$ behavior. The reparation by $\mathrm{Li}$ and $\mathrm{Gu}[30]$ is even simpler, with the same effect. They replace the velocity of sound $c$ in the eigenvalue matrix of the matrix dissipation term by $f(M) c$. Li and Gu [30] further show that a similar modification may be used in the $\mathrm{AUSM}^{+}$-up scheme, but this modification is only minor.

\subsection{The prediction step}

The prediction step is composed as follows: 
- $p_{i}^{\star}=p_{i}^{k}$

- Determination of the transporting velocity $v_{i \pm 1 / 2}^{\mathrm{T}}$ and face pressure $p_{i \pm 1 / 2}^{k}$. This is detailed in the next section.

- $\varrho_{i}^{\star}$ from the continuity equation by

$$
\begin{aligned}
\frac{1}{\Delta t}\left(\varrho_{i}^{\star}-\varrho_{i}^{n}\right)+\frac{1}{\Delta x}\left[\varrho_{i}^{\star}+\frac{1}{2} \psi_{i}\left(\varrho^{k}\right)\right] v_{i+1 / 2}^{\mathrm{T}} & \\
& -\frac{1}{\Delta x}\left[\varrho_{i-1}^{\star}+\frac{1}{2} \psi_{i-1}\left(\varrho^{k}\right)\right] v_{i-1 / 2}^{\mathrm{T}}=0
\end{aligned}
$$

- $(\varrho v)_{i}^{\star}$ from the momentum equation by

$$
\begin{aligned}
& \frac{1}{\Delta t}\left[(\varrho v)_{i}^{\star}-(\varrho v)_{i}^{n}\right]+\frac{1}{\Delta x}\left\{(\varrho v)_{i}^{\star}+\frac{1}{2} \psi_{i}\left[(\varrho v)^{k}\right]\right\} v_{i+1 / 2}^{\mathrm{T}} \\
& \quad-\frac{1}{\Delta x}\left\{(\varrho v)_{i-1}^{\star}+\frac{1}{2} \psi_{i-1}\left[(\varrho v)^{k}\right]\right\} v_{i-1 / 2}^{\mathrm{T}}+\frac{1}{\Delta x}\left(p_{i+1 / 2}^{k}-p_{i-1 / 2}^{k}\right)=0
\end{aligned}
$$

- $(\varrho E)_{i}^{\star}=\frac{p_{i}^{k}}{\gamma-1}+\frac{1}{2} \frac{\left[(\varrho v)_{i}^{\star}\right]^{2}}{\varrho_{i}^{\star}}, \quad(\varrho H)_{i}^{\star}=(\varrho E)_{i}^{\star}+p_{i}^{k}, \quad h_{i}^{\star}=\frac{\gamma}{\gamma-1} \frac{p_{i}^{k}}{\varrho_{i}^{\star}}$

\subsection{The transporting velocity and the face pressure}

The approach referred to as momentum or pressure weighted interpolation method is used to determine the transporting velocity. First, the momentum equation (2) is discretized at node $i$ as

$$
\frac{1}{\Delta t}\left[(\varrho v)_{i}^{\star \star}-(\varrho v)_{i}^{n}\right]+A_{i}^{k}(\varrho v)_{i}^{\star \star}-B_{i}^{k}+\frac{1}{\Delta x}\left(p_{i+1 / 2}^{k}-p_{i-1 / 2}^{k}\right)=0,
$$

where the notation ${ }^{\star \star}$ is used to stress that the sought transporting velocity $i$ ) is not derived from the predicted values denoted by * and $i$ ) is identical in all the conservation equations of the prediction-correction stages of the current $k$-iteration. The quantities $A_{i}^{k}$ and $B_{i}^{k}$, generated by the advection part of the equation with upwinding of the momentum, are taken at iteration level $k$ in order to obtain an explicit expression for momentum at node $i$. For the sake of clarity, the velocities appearing in these terms are called face velocities in order to distinguish them from the transporting velocities $v^{\mathrm{T}}$ introduced above. These face velocities are obtained by the $\mathrm{AUSM}^{+}$scheme (see section 3.1), namely

$$
A_{i}^{k}=\frac{1}{\Delta x} v_{i+1 / 2}^{\mathrm{AUSM}^{+}}{ }^{k}
$$




$$
B_{i}^{k}=\left\{(\varrho v)_{i-1}^{k}+\frac{1}{2} \psi_{i-1}\left[(\varrho v)^{k}\right]\right\} \frac{1}{\Delta x} v_{i-1 / 2}^{\mathrm{AUSM}^{+}}{ }^{k}-\frac{1}{2} \psi_{i}\left[(\varrho v)^{k}\right] \frac{1}{\Delta x} v_{i+1 / 2}^{\mathrm{AUSM}^{+}}
$$

Second, an equation similar to (30) is postulated at the cell-face, viz.

$$
B_{i+1 / 2}^{k}=A_{i+1 / 2}^{k}(\varrho v)_{i+1 / 2}^{\star \star}+\frac{1}{\Delta x}\left(p_{i+1}^{k}-p_{i}^{k}\right)+\frac{1}{\Delta t}\left[(\varrho v)_{i+1 / 2}^{\star \star}-(\varrho v)_{i+1 / 2}^{n}\right],
$$

with

$$
A_{i+1 / 2}^{k}=\frac{1}{2}\left(A_{i}^{k}+A_{i+1}^{k}\right), \quad B_{i+1 / 2}^{k}=\frac{1}{2}\left(B_{i}^{k}+B_{i+1}^{k}\right) .
$$

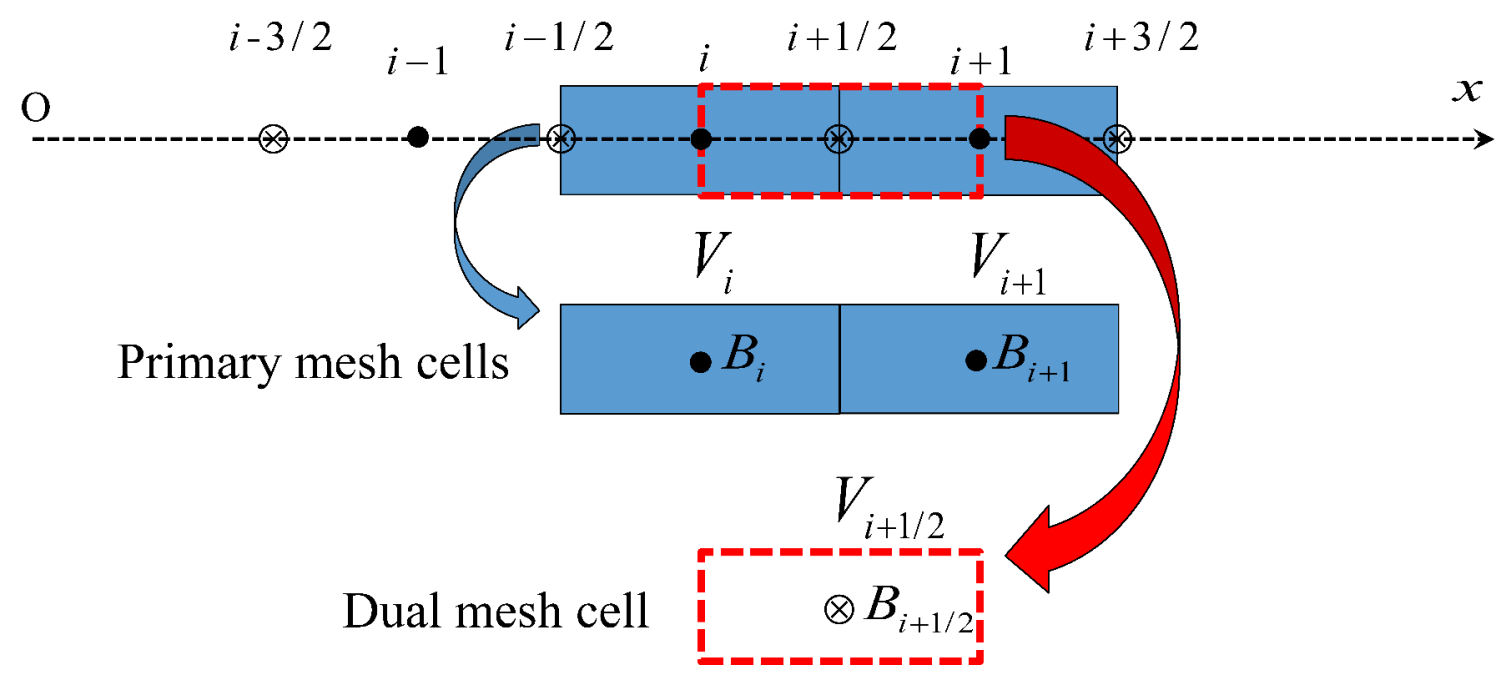

Fig. 1. Momentum interpolation stencil.

Equation (32) is a momentum equation written on a dual cell around the face center. The corresponding interpolation procedure for the $B$-terms is illustrated in Fig. 1. The consideration of the momentum equation on the dual mesh along with the interpolations of the selected groupings of terms, such as those of Eq. (33), corresponds to the momentum interpolation approach, also called averaging of the momentum equation in the following. It should be noted that no face pressure is needed in the momentum equation (32). The transporting velocity is finally obtained by

$$
v_{i+1 / 2}^{\mathrm{T}}=\frac{(\varrho v)_{i+1 / 2}^{\star \star}}{\varrho_{i+1 / 2}^{k}},
$$

where the face density is calculated as

$$
\varrho_{i+1 / 2}^{k}=\frac{1}{2}\left(\varrho_{\mathrm{L}}^{k}+\varrho_{\mathrm{R}}^{k}\right)
$$

For the face-pressure, we take (25) and (26), namely

$$
p_{i+1 / 2}^{k}=\frac{1}{2}\left(p_{\mathrm{L}}^{k}+p_{\mathrm{R}}^{k}\right)+\frac{1}{2} f_{p}^{k} \varrho_{i+1 / 2}^{k} c_{i+1 / 2}^{k}\left(v_{\mathrm{L}}^{k}-v_{\mathrm{R}}^{k}\right),
$$




$$
f_{p}^{k}=2 \widetilde{M}_{i+1 / 2}^{k}-\left(\widetilde{M}_{i+1 / 2}^{k}\right)^{2}, \quad \widetilde{M}_{i+1 / 2}^{k}=\min \left\{1, \sqrt{\frac{\left(v_{\mathrm{L}}^{k}\right)^{2}+\left(v_{\mathrm{R}}^{k}\right)^{2}}{2\left(c_{i+1 / 2}^{k}\right)^{2}}}\right\} .
$$

The scheme defined by the face transporting velocity (34) and the face pressure (36) is called the MIAU scheme, standing for Momentum Interpolation with Advection Upstream splitting scheme.

\subsection{The correction step}

The correction step is derived from the energy equation. A pure pressure correction is applied, so that the predicted value of density is taken as the value at iteration level $k+1$ :

$$
\varrho^{k+1}=\varrho^{\star} .
$$

Corrections of $\varrho v$ and $\varrho E$, $(\varrho v)^{k+1}-(\varrho v)^{\star}$ and $(\varrho E)^{k+1}-(\varrho E)^{\star}$, are linked to corrections of pressure. Corrections are denoted by a prime. With $(\varrho v)^{\prime}=(\varrho v)^{k+1}-(\varrho v)^{\star}, p^{\prime}=$ $p^{k+1}-p^{k}$ and $A_{i+1 / 2}^{k}$ defined above, we derive from (32), at a face $i+1 / 2$,

$$
(\varrho v)_{i+1 / 2}^{\prime}=-\alpha_{i+1 / 2}^{k}\left(p_{i+1}^{\prime}-p_{i}^{\prime}\right), \quad \alpha_{i+1 / 2}^{k}=\frac{\frac{1}{\Delta x}}{\frac{1}{\Delta t}+A_{i+1 / 2}^{k}} .
$$

In the energy equation, no correction is done for the kinetic energy. So

$$
(\varrho E)^{k+1}-(\varrho E)^{\star}=(\varrho e)^{k+1}-(\varrho e)^{k} \simeq\left[\partial_{p}(\varrho e)\left(p^{k}, \varrho^{\star}\right)\right]\left(p^{k+1}-p^{k}\right)=\frac{1}{\gamma-1} p^{\prime} .
$$

The flux term is written as

$$
(\varrho v H)^{k+1}=\left(\frac{1}{2} \varrho v^{2} v\right)^{\star}+(\varrho h v)^{k+1} .
$$

Corrections are introduced by

$$
(\varrho h v)^{k+1} \simeq(\varrho h v)^{\star}+v^{\star}\left[(\varrho h)^{k+1}-(\varrho h)^{\star}\right]+h^{\star}\left[(\varrho v)^{k+1}-(\varrho v)^{\star}\right],
$$

where

$$
(\varrho h)^{k+1}-(\varrho h)^{\star} \simeq \partial_{p}(\varrho h)\left(p^{k}, \varrho^{\star}\right)\left(p^{k+1}-p^{k}\right)=\frac{\gamma}{\gamma-1} p^{\prime} .
$$

Thus, the equation for pressure correction is given by

$$
\begin{aligned}
\frac{1}{\Delta t}\left[\partial_{p}(\varrho e)\left(p^{k}, \varrho^{\star}\right)\right] & \left(p^{k+1}-p^{k}\right)+\partial_{x}\left\{v^{\star}\left[\partial_{p}(\varrho h)\left(p^{k}, \varrho^{\star}\right)\left(p^{k+1}-p^{k}\right)\right]\right\} \\
+\partial_{x}\left\{h ^ { \star } \left[(\varrho v)^{k+1}\right.\right. & \left.\left.-(\varrho v)^{\star}\right]\right\} \\
& =-\left(\frac{1}{\Delta t}\left[(\varrho E)^{\star}-(\varrho E)^{n}\right]+\partial_{x}\left(\frac{1}{2} \varrho v^{2} v\right)^{\star}+\partial_{x}(\varrho h v)^{\star}\right) .
\end{aligned}
$$


It is written in compact form as

$$
C_{i-1} p_{i-1}^{\prime}+C_{i} p_{i}^{\prime}+C_{i+1} p_{i+1}^{\prime}=-\Sigma_{i},
$$

where, with the notation $\tau=\Delta t / \Delta x$,

$$
\begin{aligned}
& C_{i-1}=-\tau \frac{\gamma}{\gamma-1} v_{i-1 / 2}^{\mathrm{T}}-\tau \alpha_{i-1 / 2}^{k} h_{i-1}^{\star}, \\
& C_{i}= \frac{1}{\gamma-1}+\tau \frac{\gamma}{\gamma-1} v_{i+1 / 2}^{\mathrm{T}}+\tau\left(\alpha_{i+1 / 2}^{k} h_{i}^{\star}+\alpha_{i-1 / 2}^{k} h_{i-1}^{\star}\right), \\
& C_{i+1}=-\tau \alpha_{i+1 / 2}^{k} h_{i}^{\star}, \\
& \Sigma_{i}=\left[(\varrho E)_{i}^{\star}-(\varrho E)_{i}^{n}\right]+\tau\left\{\frac{1}{2} \frac{\left[(\varrho v)_{i}^{\star}\right]^{2}}{\varrho_{i}^{\star}}+\frac{1}{2} \psi_{i}\left(\frac{1}{2} \frac{\left[(\varrho v)^{k}\right]^{2}}{\varrho^{k}}\right)\right\} v_{i+1 / 2}^{\mathrm{T}} \\
&-\tau\left\{\frac{1}{2} \frac{\left[(\varrho v)_{i-1}^{\star}\right]^{2}}{\varrho_{i-1}^{\star}}+\frac{1}{2} \psi_{i-1}\left(\frac{1}{2} \frac{\left[(\varrho v)^{k}\right]^{2}}{\varrho^{k}}\right)\right\} v_{i-1 / 2}^{\mathrm{T}} \\
& \quad+\tau\left\{(\varrho h)_{i}^{\star}+\frac{1}{2} \psi_{i}\left[(\varrho h)^{k}\right]\right\} v_{i+1 / 2}^{\mathrm{T}}-\tau\left\{(\varrho h)_{i-1}^{\star}+\frac{1}{2} \psi_{i-1}\left[(\varrho h)^{k}\right]\right\} v_{i-1 / 2}^{\mathrm{T}} .
\end{aligned}
$$

\subsection{Updates}

Updated values are calculated by

$$
\begin{aligned}
& p_{i}^{k+1}=p_{i}^{k}+p_{i}^{\prime}, \\
& \varrho_{i}^{k+1}=\varrho_{i}^{\star}, \\
& (\varrho v)_{i}^{k+1}=(\varrho v)_{i}^{\star}+(\varrho v)_{i}^{\prime}=(\varrho v)_{i}^{\star}-\alpha_{i}^{k}\left(p_{i+1 / 2}^{\prime}-p_{i-1 / 2}^{\prime}\right), \\
& (\varrho E)_{i}^{k+1}=(\varrho E)_{i}^{\star}+\frac{1}{\gamma-1} p_{i}^{\prime},
\end{aligned}
$$

with

$$
p_{i+1 / 2}^{\prime}=\frac{1}{2}\left(p_{i}^{\prime}+p_{i+1}^{\prime}\right) \quad \text { and } \quad \alpha_{i}^{k}=\frac{\frac{1}{\Delta x}}{\frac{1}{\Delta t}+A_{i}^{k}} .
$$

\section{Comparison with other methods}

In this section, the basic options for constructing the momentum interpolation method, as element of a predictor-corrector algorithm, are reviewed. Some examples are discussed and our particular choices are motivated. 
The interpolation of the momentum equations, called the momentum interpolation, can be done in many different ways. As a consequence, any method of the literature can be singled out for some of its specific aspects. But, for the present basic classification of the different types of methods, not all differentiating aspects are discussed.

A first distinction can be made between methods with unique or non-unique transporting velocity in the convection part of the equations. The transporting velocity of the MIAU scheme described in section 3.4 is calculated at the beginning of the prediction loop, and is then used in all the equations of the prediction and correction steps. Such a choice is here referred to as using a unique transporting velocity. In such a case, for a time-dependent multi-dimensional flow, with a finite volume method and first order implicit time stepping, the momentum equation considered before the interpolation for the derivation of the transporting velocity may be written as

$$
V_{P} \frac{(\varrho v)_{j, P}^{\star \star}-(\varrho v)_{j, P}^{n}}{\Delta t}+A_{P}^{k}(\varrho v)_{j, P}^{\star \star}-\sum A_{Q}^{k}(\varrho v)_{j, Q}^{\star \star}+V_{P}\left(\partial_{x_{j}} p\right)_{P}^{k}=0
$$

where $V_{P}$ is the volume around the node $P, v_{j, P}$ is the velocity component in the $x_{j}$ direction, $Q$ indicates neighboring nodes, $k$ is the iteration level of the predictor-corrector loop aimed at obtaining the solution at time level $n+1$ from that known at time level $n$, ${ }^{\star \star}$ indicates preliminary values before the prediction step during iteration $k \rightarrow k+1$, and $A_{P}^{k}$ and $A_{Q}^{k}$ are resulting from the discretization of the flux balance of the advective (and possibly diffusive) terms.

In order to allow for an explicit determination of the transporting velocity components, $\varrho_{P}^{\star \star}$ is then replaced by $\varrho_{P}^{k}$, and $B_{P}^{\star \star}=\sum A_{Q}^{k}(\varrho v)_{j, Q}^{\star \star}$ by $B_{P}^{k}=\sum A_{Q}^{k}(\varrho v)_{j, Q}^{k}$. Dividing Eq. (45) by the cell volume then yields

$$
\frac{\varrho_{P}^{k} v_{j, P}^{\star \star}-\varrho_{P}^{n} v_{j, P}^{n}}{\Delta t}+a_{P}^{k} \varrho_{P}^{k} v_{j, P}^{\star \star}-b_{P}^{k}+\left(\partial_{x_{j}} p\right)_{P}^{k}=0 .
$$

Eqs. (46) are then interpolated to the faces of the control volumes, resulting in an expression of the unique transporting velocity at a face.

Another choice is to calculate the transporting velocity between the prediction and the correction steps. This implies that for a given $k$ iteration, the transporting velocity of the correction step will be different from that of the prediction step. This is referred to as using a non-unique transporting velocity. In such a case, the counterpart of Eqs. (45) can be written as

$$
V_{P} \frac{(\varrho v)_{j, P}^{\star}-\varrho_{P}^{n} v_{j, P}^{n}}{\Delta t}+A_{P}^{k}(\varrho v)_{j, P}^{\star}-\sum A_{Q}^{k}(\varrho v)_{j, Q}^{\star}+V_{P}\left(\partial_{x_{j}} p\right)_{P}^{k}=0
$$

where ${ }^{\star}$ indicates the predicted values during iteration $k \rightarrow k+1$. The momentum equations (47) are then interpolated to the faces of the control volumes, for construction of a second transporting velocity, or the corresponding mass flux, that is used in the pressurecorrection equation, which is derived either from the continuity equation or from the energy equation. 
From now on, further elements of the momentum interpolation are discussed for the method with a unique transporting velocity.

A second distinction between the different momentum interpolation approaches comes from the grouping of the terms before interpolation. With the grouping originally proposed by Rhie and Chow [9] for steady constant-density flows, the momentum equation (46) simplifies into

$$
\left[a_{P}^{k} \varrho\right] v_{j, P}^{\star \star}-\left[b_{P}^{k}\right]+\left(\partial_{x_{j}} p\right)_{P}^{k}=0,
$$

where the subsequent interpolation concerns the terms in square brackets. The extension to the time-dependent equations (46) can be done in different ways depending on the kind of groupings selected. Two examples are

$$
\begin{aligned}
& {\left[\frac{\varrho_{P}^{k}}{\Delta t}+a_{P}^{k} \varrho_{P}^{k}\right] v_{j, P}^{\star \star}-\left[\frac{\varrho_{P}^{n}}{\Delta t} v_{j, P}^{n}+b_{P}^{k}\right]+\left(\partial_{x_{j}} p\right)_{P}^{k}=0} \\
& {\left[\varrho_{P}^{k}\right]\left[\frac{1}{\Delta t}+a_{P}^{k}\right] v_{j, P}^{\star \star}-\left[\varrho_{P}^{n}\right] \frac{1}{\Delta t} v_{j, P}^{n}-\left[b_{P}^{k}\right]+\left(\partial_{x_{j}} p\right)_{P}^{k}=0 .}
\end{aligned}
$$

The transporting velocity components are then obtained by averaging the momentum equations i.e. writing them on the dual mesh and interpolating the terms in square brackets. The pressure gradient is discretized in compact form across the face. With Eq. (49), the formulation is the simplest since there is no time stepping left. This leads to a steady state solution (if any) which depends on the size of the time step. With Eq. (50), the time stepping of the transporting velocity components remains and a steady state solution does not depend on the size of the time step. The MIAU method that we propose uses the form (50). Along with a steady state that is time-step independent, it is fully time-consistent in the sense that the time-derivative term of the velocity and the pressure gradient term are written locally at the face in the interpolated momentum equation. For a flow at very low speed, thus for vanishing convection (and diffusion), thus terms $a_{P}^{k}$ and $b_{P}^{k}$ very near to zero, the interpolated momentum equation expresses then the local equilibrium between inertia (with an averaged density) and pressure gradient. This is essential for correct representation of the propagation speed of acoustic waves in a low Mach number flow, as we already mentioned in section 2.6.

A third distinction is done according to the chosen normalization of the momentum equations. Dividing Eq. (45) by the volume of the cell is already a way of normalization, but for a global classification, no distinction is made here between the forms (45) and (46). In the original method of Rhie and Chow, Eq. (48) is divided by the coefficient $\left(a_{P}^{k} \varrho\right)$ into

$$
v_{j, P}^{\star \star}-\left[\frac{b_{P}^{k}}{a_{P}^{k} \varrho}\right]+\left[\frac{1}{a_{P}^{k} \varrho}\right]\left(\partial_{x_{j}} p\right)_{P}^{k}=0 .
$$

With respect to the normalization of the momentum equations, we may distinguish undivided equations (Eqs. (49) and (50)) and Rhie-Chow normalized equations, obtained by dividing the equations by $\left(a_{P}^{k} \varrho_{P}^{k}\right)$. But other normalizations are possible. 
A fourth criterion is related to the discretization of the convection part of the equations and the pressure gradient in the momentum equation used to determine the transporting velocity. In many methods, the face velocity and the face pressure therein are obtained by linear interpolation, combined with an upwind discretization of the convection parts. An alternative is to use specific upwind methods for good representation of discontinuities in high Mach number flows, as the flux-vector splitting methods AUSM $[18,19]$ or SLAU $[27,28]$.

Many methods are not time-consistent and thus generate a steady state that is time-step dependent. A recent example of this class of methods is that of $\mathrm{Li}$ and $\mathrm{Gu}$ [32]. The transporting velocity is the same in all equations and is obtained from a preliminary discretization of the momentum equations where the face values are obtained by linear interpolation. In the final discretization, the face pressure is also a linearly interpolated value. This is justified for low Mach number flows, but it may create oscillations around shocks in high Mach number flows (not tested). Thus, for high speed flows, a Mach number dependent interpolation between the proposed method and the flux-difference method by Roe is suggested. So, the basic method of Li and Gu [32] is not a genuine all-Mach number method.

Many methods are time-consistent, but do not have a unique transporting velocity. We cite the methods by Lien and Leschziner [12], Shen et al. [33] and two methods by Pascau [13].

As a recent example, we discuss the method of Ong and Chan [14]. The upwinding of the advection part is done with a Rusanov flux: the flux of a transported quantity is the face volume flux multiplied with the average of the values on both face sides of the transported quantity per unit of volume minus the absolute value of the face mass flux multiplied with half of the difference on both face sides of the transported quantity per unit of mass. This way of upwinding differs from ours, but this is a rather technical aspect and not a fundamental difference. The mass flux is SLAU2 [28], but without the pressure gradient term. The volume flux is defined with a similar expression (density terms left out). The pressure flux is SLAU2 without the velocity diffusive term (similar to $\mathrm{AUSM}^{+}$). So, the pressure stabilization term is left out. The pressure-velocity coupling comes from momentum interpolation. A time-consistent momentum interpolation is used for definition of a mass flux that is inserted into the continuity equation for construction of a time-dependent Poissontype equation for pressure. This means that the mass flux in the continuity equation differs from the mass flux in the momentum and energy equations. Good results are obtained for steady flows at low and high Mach number, and also good results for a low Mach number Riemann problem with only shocks. The method very likely fails for stationary or slowly moving contact discontinuities (not tested). It is also very likely that the method is rather dissipative for propagation of acoustic perturbations in low Mach number flows (not tested). For such problems, strict satisfaction of the conservation laws with a face velocity that is the same in all equations is a guarantee for the correct representation of the propagation velocity. Therefore, we opt in our method for a unique transporting velocity.

The method that we propose is quite near to the one by Denner and van Wachem [34], 
Xiao et al. [15], Bartholomew et al. [35] and Denner [36], hereafter referred to as Denner et al.. A first difference is the Rhie-Chow normalization of the momentum equations in the method of Denner et al., while we use undivided equations. But this is a rather technical aspect. A more fundamental difference it that the momentum interpolation by Denner et al. is cast into a difference form, while we use a direct form. This is an aspect that we did not discuss yet. With the original method of Rhie and Chow, using Eq. (51), the transporting velocity is

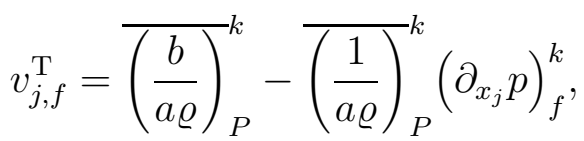

where the overbar denotes a linearly interpolated value. The expression may be compared to a linearly averaged form of the nodal equations (51) by

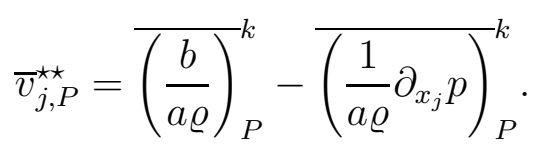

With $v_{j, P}^{\star \star}$ taken at level $k$, the difference of both expressions is

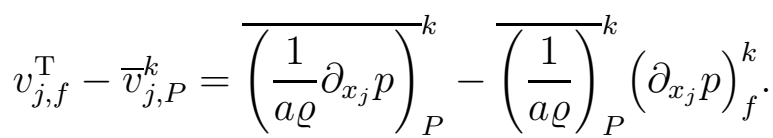

An approximate form of this expression is

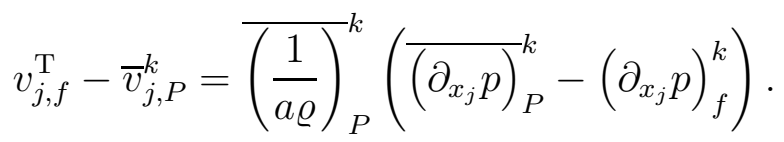

The difference formulation (54), or (55), is often used in methods with a non-unique face transporting velocity, because it yields a simple expression of the face velocity to be inserted in the equation which determines the pressure, after the prediction step for velocity $\left(\bar{v}_{j, P}^{\star}\right.$ is then used instead of $\left.\bar{v}_{j, P}^{k}\right)$. Examples are the methods by Lien and Leschziner [12], Pascau [13], and $\mathrm{Li}$ and $\mathrm{Gu}[32]$.

In our method, the face transporting velocity comes from the averaged form of Eq. (50) as

$$
\bar{\varrho}_{P}^{k}\left(\frac{1}{\Delta t}+\bar{a}_{P}^{k}\right) v_{j, f}^{\mathrm{T}}=\bar{\varrho}_{P}^{n} \frac{1}{\Delta t} v_{j, f}^{n}+\bar{b}_{P}^{k}-\left(\partial_{x_{j}} p\right)_{f}^{k} .
$$

The method of Denner et al. starts from the Rhie-Chow normalized form of Eq. (50):

$$
\left\{1+\frac{1}{\Delta t}\left[\varrho_{P}^{k}\right]\left[\frac{1}{\varrho_{P}^{k} a_{P}^{k}}\right]\right\} v_{j, P}^{\star \star}=\left[\frac{b_{P}^{k}}{\varrho_{P}^{k} a_{P}^{k}}\right]+\frac{1}{\Delta t}\left[\varrho_{P}^{n}\right]\left[\frac{1}{\varrho_{P}^{k} a_{P}^{k}}\right] v_{j, P}^{n}-\left[\frac{1}{\varrho_{P}^{k} a_{P}^{k}}\right]\left(\partial_{x_{j}} p\right)_{P}^{k} .
$$

By direct averaging, the transporting velocity is

$$
\left(1+\frac{1}{\Delta t} \bar{\varrho}_{P}^{k} d_{f}^{k}\right) v_{j, f}^{\mathrm{T}}=g_{f}^{k}+\frac{1}{\Delta t} \bar{\varrho}_{P}^{n} d_{f}^{k} v_{j, f}^{n}-d_{f}^{k}\left(\partial_{x_{j}} p\right)_{f}^{k},
$$




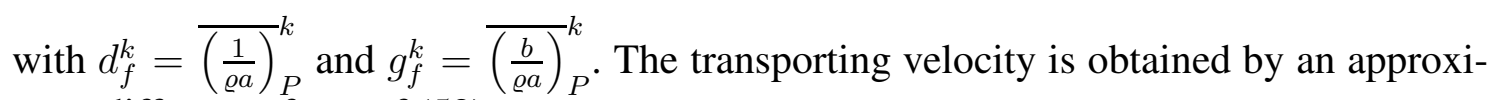
mate difference form of (58) as

$$
\left(1+\frac{1}{\Delta t} \bar{\varrho}_{P}^{k} d_{f}^{k}\right)\left(v_{j, f}^{\mathrm{T}}-\bar{v}_{j, P}^{k}\right)=\frac{1}{\Delta t} \bar{\varrho}_{P}^{n} d_{f}^{k}\left(v_{j, f}^{n}-\bar{v}_{j, P}^{k}\right)-d_{f}^{k}\left\{\left(\partial_{x_{j}} p\right)_{f}^{k}-\overline{\left(\partial_{x_{j}} p\right)_{P}^{k}}\right\} .
$$

For large changes of density, as in two-phase flows, a variant is used:

$$
\left(1+\frac{1}{\Delta t} \varrho_{f}^{k} d_{f}^{k}\right)\left(v_{j, f}^{\mathrm{T}}-\bar{v}_{j, P}^{k}\right)=\frac{1}{\Delta t} \varrho_{f}^{n} d_{f}^{k}\left(v_{j, f}^{n}-\bar{v}_{j, P}^{k}\right)-d_{f}^{k}\left\{\left(\partial_{x_{j}} p\right)_{f}^{k}-\varrho_{f}^{k} \overline{\left(\frac{1}{\varrho} \partial_{x_{j}} p\right)_{P}^{k}}\right\},
$$

where $\varrho_{f}^{k}$ is the inverse of the linearly interpolated value of $1 / \varrho$ (harmonic average).

In the final discretization, the face pressure is a linearly interpolated value. The solution technique is fully coupled, but a pressure-correction solution is possible. The method functions well for steady flows at low and high Mach numbers and for propagation of acoustic waves in low Mach number flows. It functions also well for high Mach number Riemann problems, but there are sometimes oscillations around shocks. Very likely, these are caused by the linearly interpolated value of the face velocity in the preliminary discretization of the momentum equations and the linearly interpolated value of the pressure in the final discretization. Further, we fear that an approximate difference form of the momentum interpolation may cause some dispersion. Therefore, a direct interpolation of the momentum equations along with face velocity and face pressure derived from an AUSM-type method are adopted in the present MIAU scheme.

\section{Tests of stationary discontinuities}

Here and in the following sections, the fluid is air with $\gamma=1.4$. The time integration is carried out over the time interval $\left[0, t_{\mathrm{f}}\right]$. No more than five $k$-iterations per time-step are allowed, as will be justified later. For all one-dimensional tests, the computational domain is divided into $N$ cells of equal length. For stationary discontinuities and Riemann problems, the computational domain is the interval $[0,1](\mathrm{m})$, the initial discontinuity is located at $0.5 \mathrm{~m}$, and first-order backward differencing is used for time integration.

In this section, we present test cases to verify the representation of a steady contact discontinuity and a steady shock.

\subsection{Stationary contact discontinuity}

For this zero Mach number test, we take $N=200$ cells and $\mathrm{CFL}\left(c_{\mathrm{L}}\right)=0.5$. The initial data are listed in Table 1. 


\begin{tabular}{|c|c|c||c|c|c||c|}
\hline$\varrho_{\mathrm{L}}\left(\mathrm{kg} / \mathrm{m}^{3}\right)$ & $v_{\mathrm{L}}(\mathrm{m} / \mathrm{s})$ & $p_{\mathrm{L}}(\mathrm{Pa})$ & $\varrho_{\mathrm{R}}\left(\mathrm{kg} / \mathrm{m}^{3}\right)$ & $v_{\mathrm{R}}(\mathrm{m} / \mathrm{s})$ & $p_{\mathrm{R}}(\mathrm{Pa})$ & $t_{\mathrm{f}}(\mathrm{s})$ \\
\hline 1.4 & 0 & 1 & 1.0 & 0 & 1 & 100 \\
\hline
\end{tabular}

Table 1

Settings for the stationary contact discontinuity test of Sec. 5.1.
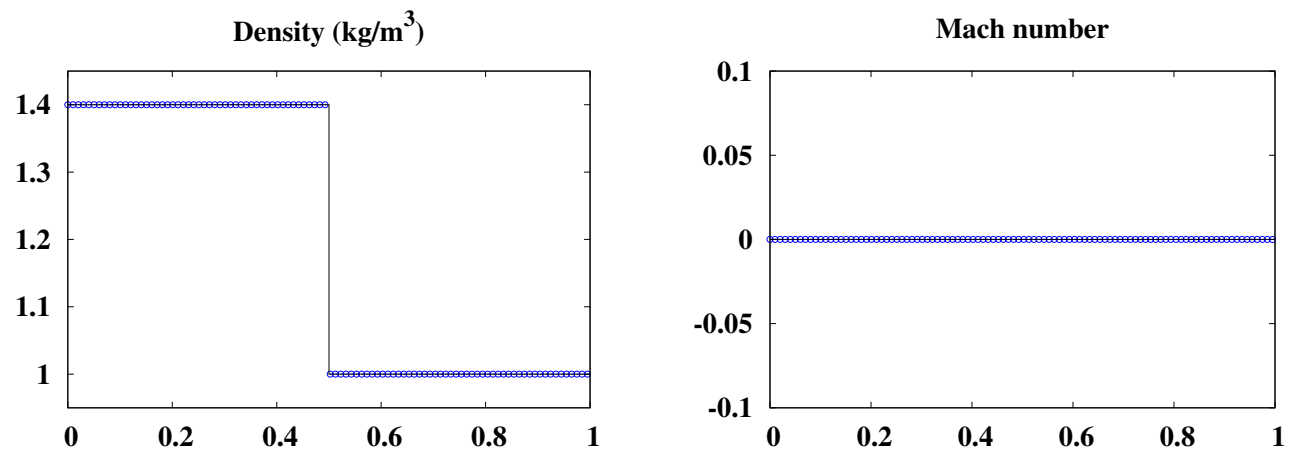

Fig. 2. Stationary contact discontinuity: MIAU scheme.

The representation of a steady contact discontinuity is perfect; see Fig. 2. That such a discontinuity is an exact solution to the method may be verified analytically.

\subsection{Stationary shock}

This test allows to check the ability of the method to satisfy the shock relations. We take $N=1000$ cells and CFL $\left(v_{\mathrm{L}}\right)=0.5$. The initial data are listed in Table 2 .

\begin{tabular}{|c|c|c||c|c|c||c|}
\hline$\varrho_{\mathrm{L}}\left(\mathrm{kg} / \mathrm{m}^{3}\right)$ & $v_{\mathrm{L}}(\mathrm{m} / \mathrm{s})$ & $p_{\mathrm{L}}(\mathrm{Pa})$ & $\varrho_{\mathrm{R}}\left(\mathrm{kg} / \mathrm{m}^{3}\right)$ & $v_{\mathrm{R}}(\mathrm{m} / \mathrm{s})$ & $p_{\mathrm{R}}(\mathrm{Pa})$ & $t_{\mathrm{f}}(\mathrm{s})$ \\
\hline 1.00 & 1000.00 & 100000.0 & 3.53 & 283.33 & 816666.7 & 0.05 \\
\hline
\end{tabular}

Table 2

Settings for the stationary shock test of Sec. 5.2.
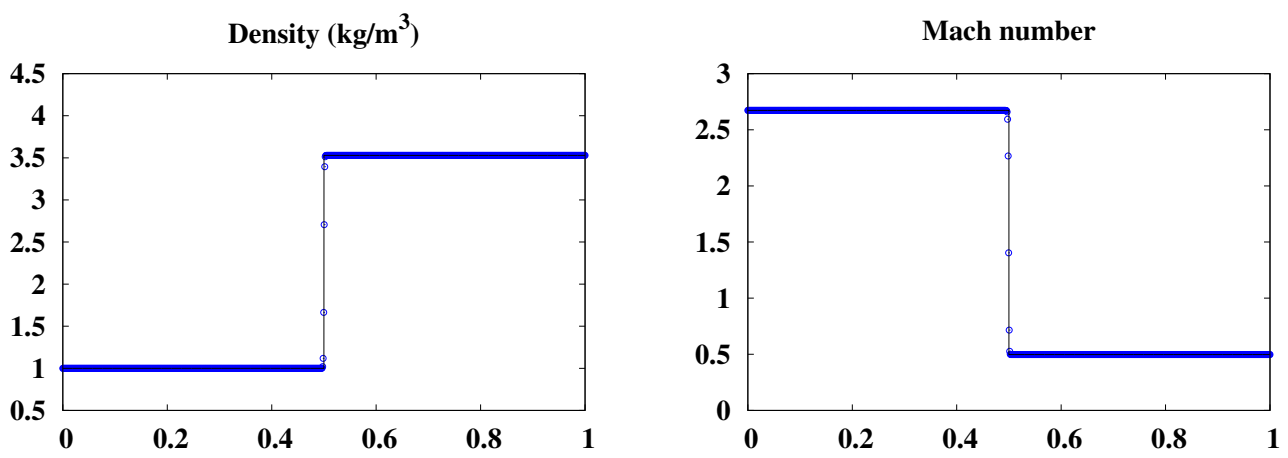

Fig. 3. Stationary shock: MIAU scheme.

The results are shown in Fig. 3. A stationary shock is represented by the MIAU scheme 
with intermediate states. This is different if the $\mathrm{AUSM}^{+}$scheme is used instead of the MIAU scheme to calculate the transporting velocity $v^{\mathrm{T}}$, since the $\mathrm{AUSM}^{+}$scheme represents a shock without intermediate states. For flows that are supersonic on one side of a face and subsonic on the other side, with flow parameters that satisfy constant stagnation enthalpy $\left(c_{\mathrm{L}}^{*}=c_{\mathrm{R}}^{*}=c^{*}\right)$ and the Prandtl relation for normal shocks $\left(v_{\mathrm{L}} v_{\mathrm{R}}=\left(c^{*}\right)^{2}\right)$, the $\mathrm{AUSM}^{+}$formulae generate face velocity and face pressure equal to the values of the supersonic state. Together with the upwinding of density, this means that the face state is equal to the supersonic state. By this property, a normal shock is preserved by the $\mathrm{AUSM}^{+}$formulae without generation of intermediate states. This property is lost with the MIAU scheme because the face values of velocity by (32)-(35), and pressure by (36)-(37), become values in between the nodal values. The consequence is the presence of intermediate states in the captured shock. But, by the conservative discretization of the equations, the shock relations are satisfied between states somewhat farther from the position of the shock, as demonstrated by Fig. 3. There are no oscillations around the shocks because the face values of velocity and pressure are in between the nodal values. It can be seen in Fig. 4 that results deteriorate when using a linear interpolation instead of the MIAU expression for the face pressure.
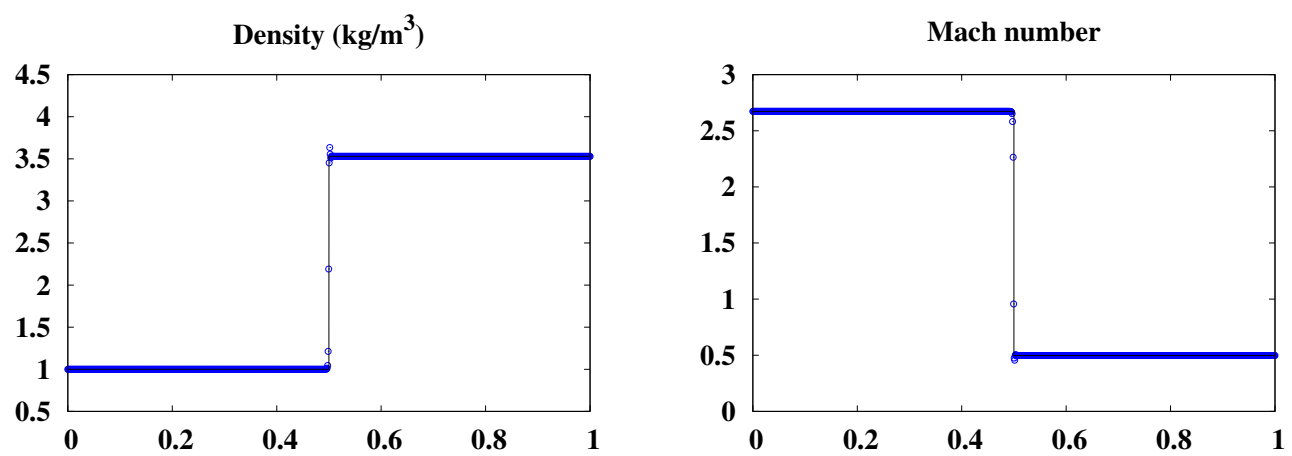

Fig. 4. Stationary shock. Flux scheme: MIAU face velocity and linear interpolation for the face pressure.

\section{Test of a low Mach number flow in the hydrodynamic limit}

Here, the ability of the method to address the hydrodynamic low Mach number limit of the Euler equations is checked.

A quasi-one-dimensional flow in a nozzle with variable cross-section area $S$ is considered. The Euler equations read

$$
\begin{aligned}
& \partial_{t}(\varrho S)+\partial_{x}(\varrho v S)=0, \\
& \partial_{t}(\varrho v S)+\partial_{x}[(\varrho v v+p) S]=p \mathrm{~d}_{x} S, \\
& \partial_{t}(\varrho E S)+\partial_{x}(\varrho v H S)=0, \\
& E=e+\frac{1}{2} v^{2}, \varrho H=\varrho E+p, \varrho e=\frac{p}{\gamma-1} .
\end{aligned}
$$


The discretization is the straightforward extension for variable cross-section area of the method detailed in section 3 . The source term in the momentum equation is discretized by $p_{i}\left(S_{i+1 / 2}-S_{i-1 / 2}\right) / \Delta x$. The corresponding source term in the interpolated momentum equation is $p_{i+1 / 2}\left(S_{i+1}-S_{i}\right)$.

We consider a one-meter long converging-diverging nozzle with

$$
S(x)= \begin{cases}0.1, & 0 \leq x \leq 0.1, \\ 0.1\left\{0.1+0.9\left[2\left(\frac{x-0.4}{0.3}\right)^{2}-\left(\frac{x-0.4}{0.3}\right)^{4}\right]\right\}, & 0.1 \leq x \leq 0.7, \\ 0.1, & 0.7 \leq x \leq 1,\end{cases}
$$

where the dimensions are in meter. The nozzle is divided into $N=400$ cells of equal length. The density and velocity at the inlet $x=0$ are $1.2046 \mathrm{~kg} / \mathrm{m}^{3}$ and $0.030886 \mathrm{~m} / \mathrm{s}$, respectively. The pressure at the outlet $x=1 \mathrm{~m}$ is $101300 \mathrm{~Pa}$. The solution is initialized with the steady zero Mach number solution for the nozzle. We take CFL $(v+c)=100$ with $v+c$ calculated with the boundary values. The duration of the simulation is $t_{f}=400 \mathrm{~s}$.

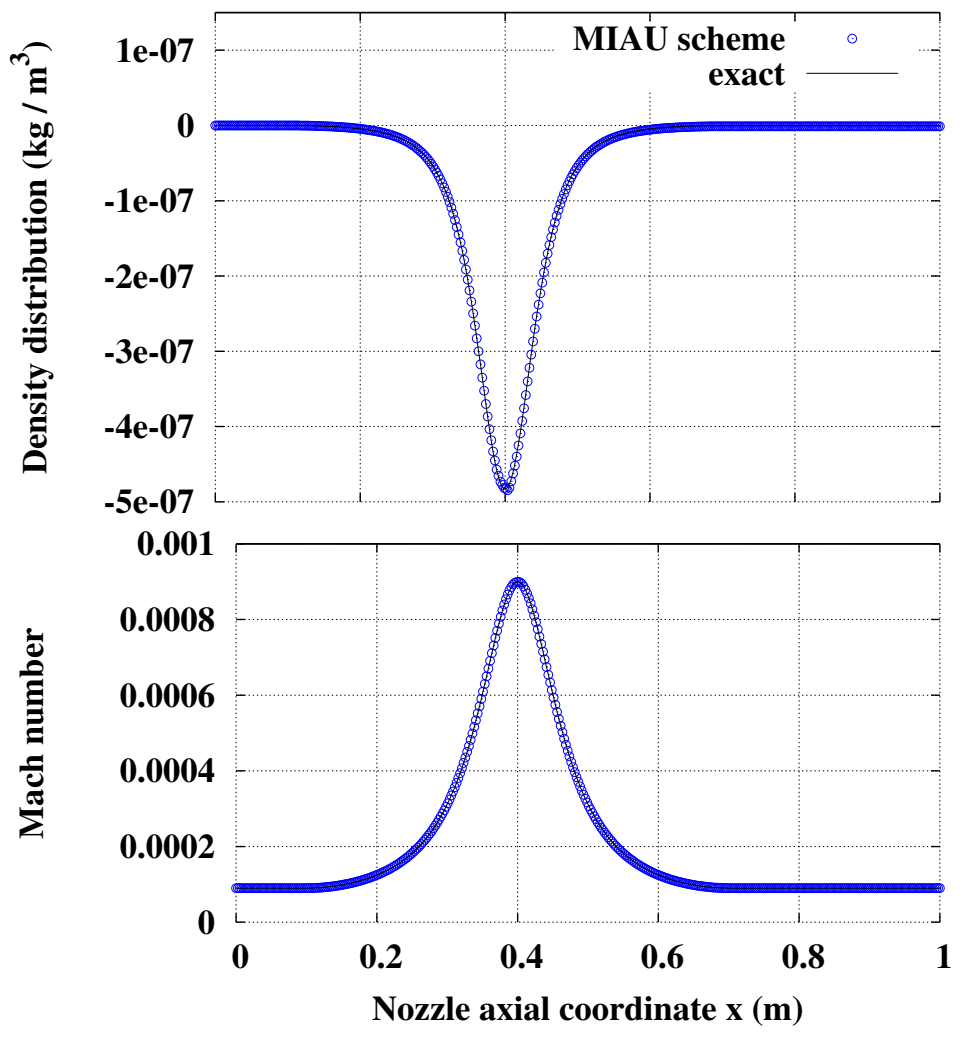

Fig. 5. Low Mach number steady nozzle flow: MIAU scheme.

The good representation of the steady hydrodynamic solution for this test (see Fig. 5) demonstrates the suitability of the $f_{p}$ factor in Eq. (36). We remark that with $f_{p}=1$, the convergence is lost. 


\section{Tests of Riemann problems}

The good functioning of the MIAU scheme for Riemann problems at low and high Mach number is now demonstrated. Results of the proposed scheme are also compared with those of the $\mathrm{AUSM}^{+}$and $\mathrm{AUSM}^{+}$-up schemes, which are detailed in Appendix A. The $\mathrm{AUSM}^{+}$-up scheme is used with the typical values of its parameters, $\sigma=1, K_{v}=0.75$ and $K_{p}=0.25$. The time integration is first order backward differencing (BDF1), but in the first test case, results with a second order backward differencing (BDF2) are also presented.

\subsection{Moving contact discontinuity}

It was shown in section 5.1 that the MIAU scheme is able to compute a stationary contact discontinuity. Because the face velocity expression of the MIAU scheme features an inertia term, it is enlightening to consider the test of a moving contact dicontinuity, proposed by van der Heul et al. [37] (see also Xiao [38]). For this test, $N=400$ cells, $\operatorname{CFL}\left(v_{\mathrm{L}}\right)=0.4$ and the initial data are listed in Table 3. As shown in Fig. 6, good agreement between exact and computed profiles is obtained.

\begin{tabular}{|c|c|c||c|c|c||c|}
\hline$\varrho_{\mathrm{L}}\left(\mathrm{kg} / \mathrm{m}^{3}\right)$ & $v_{\mathrm{L}}(\mathrm{m} / \mathrm{s})$ & $p_{\mathrm{L}}(\mathrm{Pa})$ & $\varrho_{\mathrm{R}}\left(\mathrm{kg} / \mathrm{m}^{3}\right)$ & $v_{\mathrm{R}}(\mathrm{m} / \mathrm{s})$ & $p_{\mathrm{R}}(\mathrm{Pa})$ & $t_{\mathrm{f}}(\mathrm{s})$ \\
\hline 1 & 0.5 & 0.5 & 0.5 & 0.5 & 0.5 & 0.3 \\
\hline
\end{tabular}

Table 3

Settings for the moving discontinuity of Sec. 7.1.
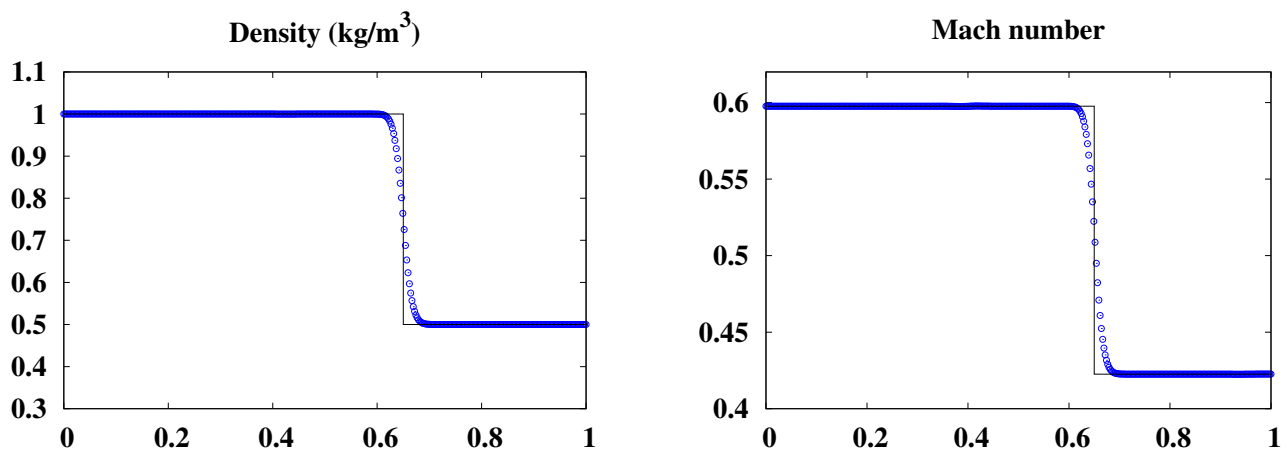

Fig. 6. Moving contact discontinuity: MIAU scheme.

The space discretization is of second order and the time integration is of first order (BDF1). But, in the vicinity of discontinuities, there is some loss of accuracy. Fig. 7 displays the convergence rate of the 1-norm of the errors of density, velocity and pressure obtained by refining the grid keeping constant the CFL number. The convergence rate is about 1.1 for velocity and pressure and about $1 / 2$ for density. The results obtained with a second order backward differencing (BDF2) are shown in Fig. 8. The convergence rate is approximately 1.2 for velocity and pressure and about $2 / 3$ for density. The convergence 
rates of $1 / 2$ and $2 / 3$ for the (discontinuous) density are in agreement with the analysis of Banks et al. [39].

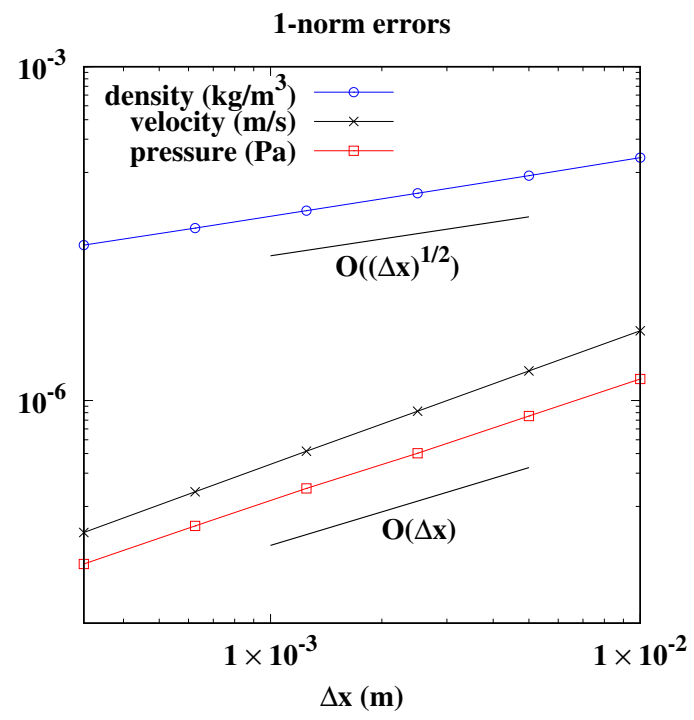

Fig. 7. Moving contact discontinuity: MIAU scheme and BDF1 time integration. Convergence rates of the 1-norm of the errors. $\mathrm{CFL}=0.8$.

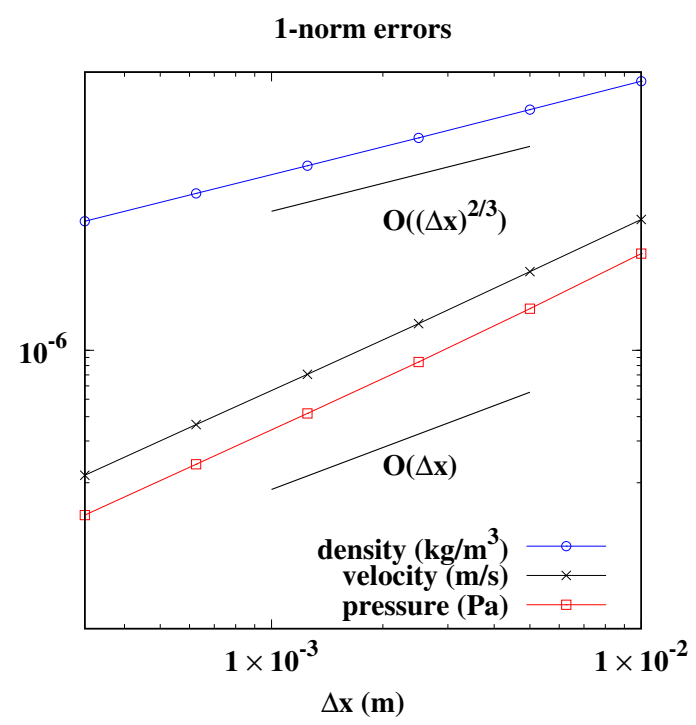

Fig. 8. Moving contact discontinuity: MIAU scheme and BDF2 time integration. Convergence rates of the 1-norm of the errors. $\mathrm{CFL}=0.4$.

\subsection{Modified Sod test}

This test, proposed by Toro [2], allows to check the entropy property and the ability of the method to capture the transport velocities of shocks, contact discontinuities and rarefaction waves. We take $N=1000$ cells and $\operatorname{CFL}\left(v_{\mathrm{L}}\right)=0.5$. The initial data are listed in Table 4. 


\begin{tabular}{|c|c|c||c|c|c||c|}
\hline$\varrho_{\mathrm{L}}\left(\mathrm{kg} / \mathrm{m}^{3}\right)$ & $v_{\mathrm{L}}(\mathrm{m} / \mathrm{s})$ & $p_{\mathrm{L}}(\mathrm{Pa})$ & $\varrho_{\mathrm{R}}\left(\mathrm{kg} / \mathrm{m}^{3}\right)$ & $v_{\mathrm{R}}(\mathrm{m} / \mathrm{s})$ & $p_{\mathrm{R}}(\mathrm{Pa})$ & $t_{\mathrm{f}}(\mathrm{s})$ \\
\hline 1 & 0.75 & 1 & 0.125 & 0 & 0.1 & 0.2 \\
\hline
\end{tabular}

Table 4

Settings for the modified Sod test of Sec. 7.2.
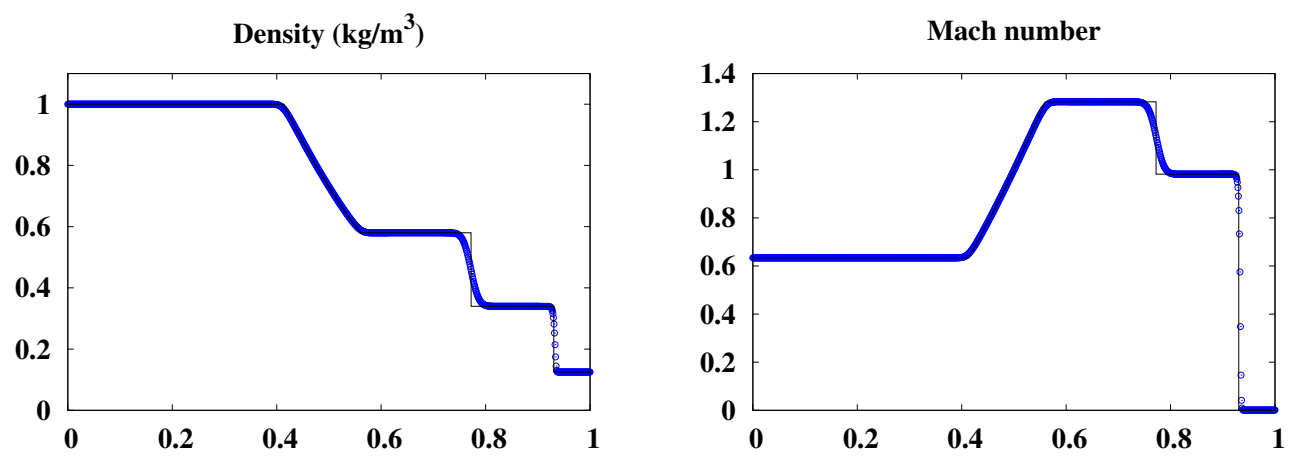

Fig. 9. Modified Sod test: MIAU scheme.

The good results obtained with the MIAU scheme are illustrated in Fig. 9. The AUSM ${ }^{+}$ and $\mathrm{AUSM}^{+}$-up schemes, used instead of the MIAU scheme to calculate the transporting velocity $v^{\mathrm{T}}$, function also well for this test (not shown).

\subsection{Low Mach number Riemann problem}

This test is proposed in [8]; see [40] for a theoretical analysis. We take $N=1000$ cells and $\operatorname{CFL}\left(v_{\mathrm{L}}+c_{\mathrm{L}}\right)=0.5$. The initial data are listed in Table 5 .

\begin{tabular}{|c|c|c||c|c|c||c|}
\hline$\varrho_{\mathrm{L}}\left(\mathrm{kg} / \mathrm{m}^{3}\right)$ & $v_{\mathrm{L}}(\mathrm{m} / \mathrm{s})$ & $p_{\mathrm{L}}(\mathrm{Pa})$ & $\varrho_{\mathrm{R}}\left(\mathrm{kg} / \mathrm{m}^{3}\right)$ & $v_{\mathrm{R}}(\mathrm{m} / \mathrm{s})$ & $p_{\mathrm{R}}(\mathrm{Pa})$ & $t_{\mathrm{f}}(\mathrm{s})$ \\
\hline 25 & 0.200 & 10000.00 & 25 & 0.202 & 10000.85 & 0.01 \\
\hline
\end{tabular}

Table 5

Settings for the low Mach number Riemann problem of Sec. 7.3.
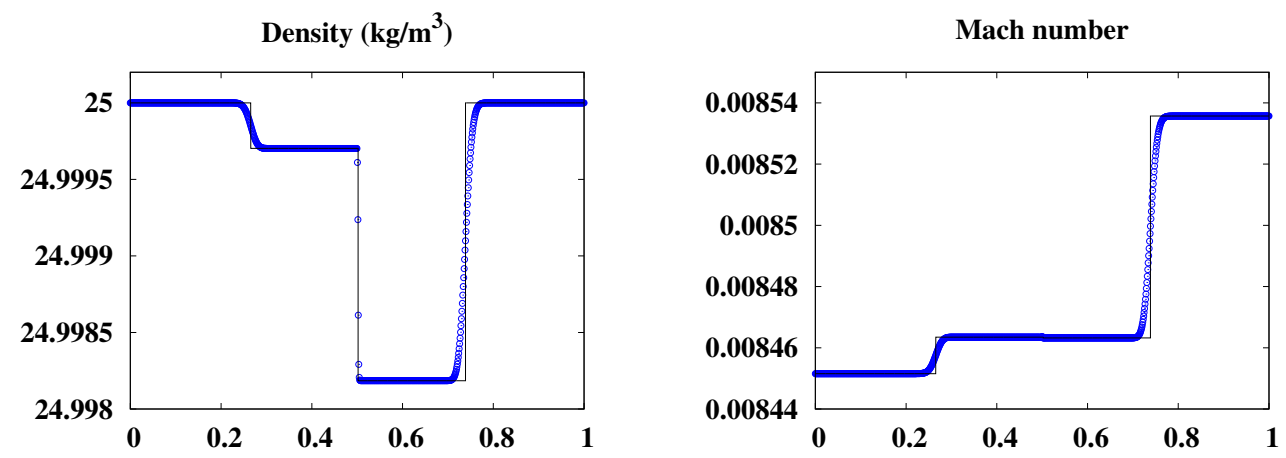

Fig. 10. Low Mach number Riemann problem: MIAU scheme. $\operatorname{CFL}\left(v_{\mathrm{L}}+c_{\mathrm{L}}\right)=0.5$. 
The good result by the MIAU scheme is shown in Fig. 10. As illustrated by Figs. 11 and 12 , the $\mathrm{AUSM}^{+}$scheme does not function for a low Mach number Riemann problem, but the $\mathrm{AUSM}^{+}$-up scheme gives a good result.
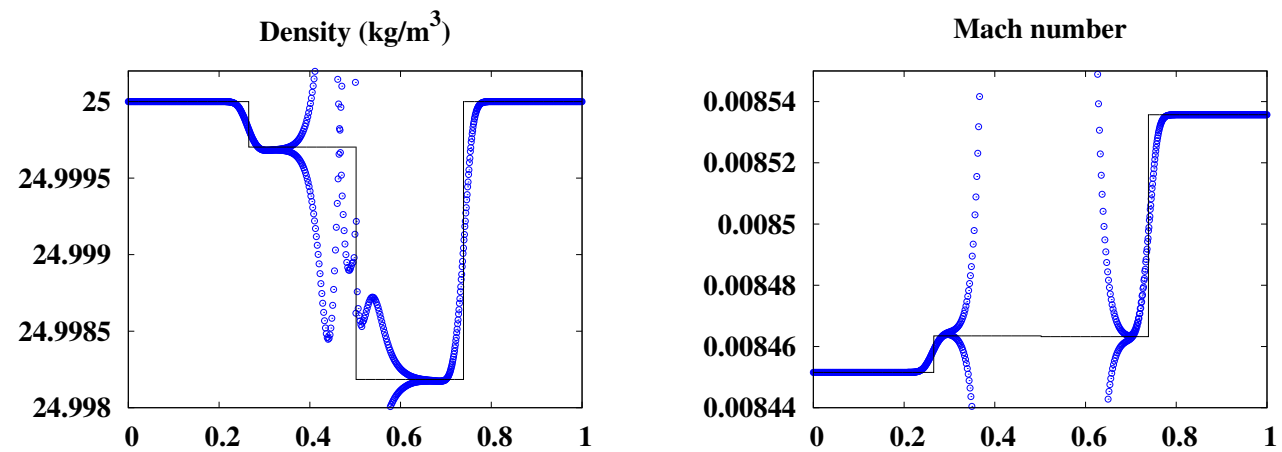

Fig. 11. Low Mach number Riemann problem: $\operatorname{AUSM}^{+}$scheme. CFL $\left(v_{\mathrm{L}}+c_{\mathrm{L}}\right)=0.5$.
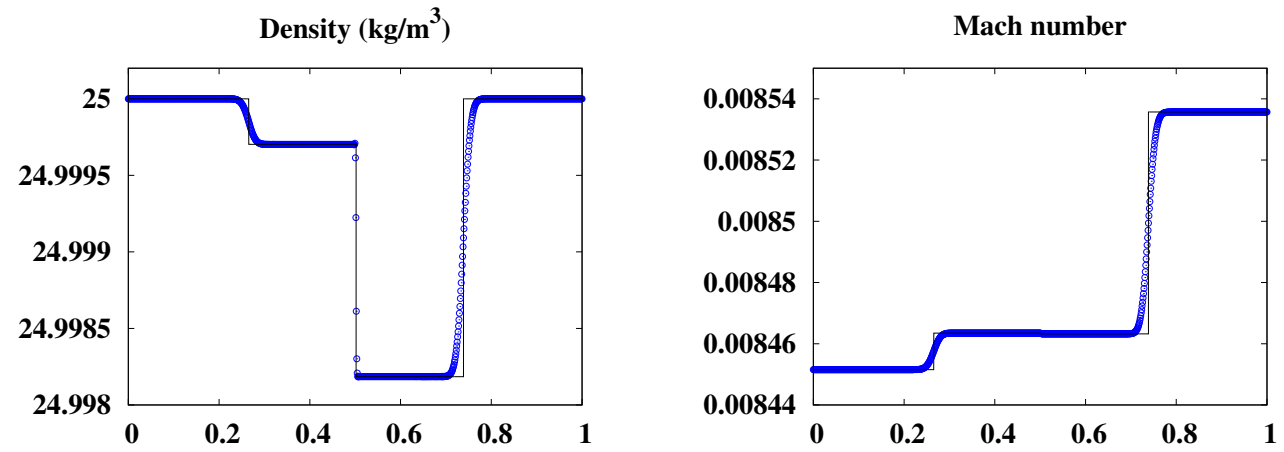

Fig. 12. Low Mach number Riemann problem: $\mathrm{AUSM}^{+}$-up scheme with $f_{c}\left(M_{0}\right)=M_{0}\left(2-M_{0}\right)$ and $M_{\infty}=10^{-2} . \operatorname{CFL}\left(v_{\mathrm{L}}+c_{\mathrm{L}}\right)=0.5$.
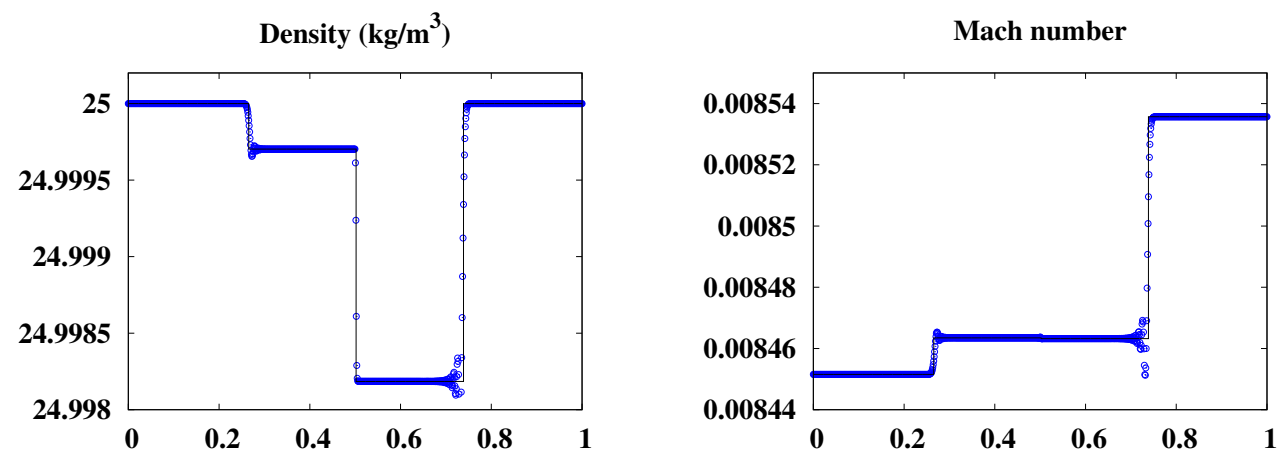

Fig. 13. Low Mach number Riemann problem: MIAU scheme. $\operatorname{CFL}\left(v_{\mathrm{L}}+c_{\mathrm{L}}\right)=10^{-2}$.

The result by the MIAU scheme for a low value of the CFL number $\left(\operatorname{CFL}\left(v_{\mathrm{L}}+c_{\mathrm{L}}\right)=\right.$ $10^{-2}$ ) is shown in Fig. 13. Oscillations are obtained. The objective of the momentum interpolation (32) is to connect the face velocity to the pressure difference accross the face. Clearly, this connection is lost for a very low value of the time-step. So, the use of the present momentum interpolation with very low values of the time-step has to be further investigated. 


\subsection{Low density flow}

This test, proposed by Toro [2], allows to check the ability of the method to correctly capture a low density flow, which occurs here between the two rarefaction waves of the solution. We take $N=1000$ cells, and $\operatorname{CFL}\left(c_{\mathrm{L}}=c_{\mathrm{R}}\right)=0.5$. The duration is $t_{\mathrm{f}}=0.15 \mathrm{~s}$ and the initial conditions are

$$
\left(\varrho\left(\mathrm{kg} / \mathrm{m}^{3}\right), v(\mathrm{~m} / \mathrm{s}), p(\mathrm{~Pa})\right)= \begin{cases}(1,-2,0.4), & x \leq 0.5 \mathrm{~m}, \\ (1,2,0.4), & x>0.5 \mathrm{~m} .\end{cases}
$$

We observed that this test does not succeed when the pressure correction method is used with all the limiters presented in section 3. This failure is also observed with the AUSM ${ }^{+}$ and $\mathrm{AUSM}^{+}$-up schemes. However, with first-order upwind space discretization for density and momentum in the prediction step, and kinetic energy in the RHS of the energy equation in the correction step (see section 3), good results are obtained with the MIAU scheme, as shown in Figure 14. The $\mathrm{AUSM}^{+}$and $\mathrm{AUSM}^{+}$-up schemes function also well for this case (not shown).
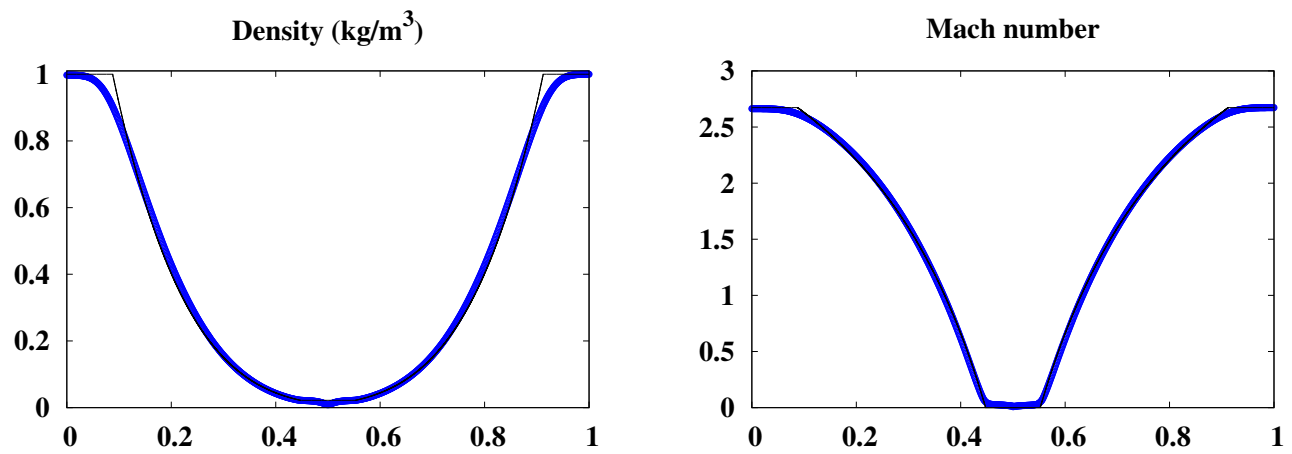

Fig. 14. Low density flow: MIAU scheme.

\subsection{Mach 3 shock}

This test is proposed in [41]; see also [38]. We take $N=1000$ cells and $\operatorname{CFL}\left(v_{\mathrm{L}}\right)=0.5$. The initial data are listed in Table 6. Satisfactory results are obtained, as shown in Fig. 15. The $\mathrm{AUSM}^{+}$and $\mathrm{AUSM}^{+}$-up schemes function also well for this case (not shown).

\begin{tabular}{|c|c|c||c|c|c||c|}
\hline$\varrho_{\mathrm{L}}\left(\mathrm{kg} / \mathrm{m}^{3}\right)$ & $v_{\mathrm{L}}(\mathrm{m} / \mathrm{s})$ & $p_{\mathrm{L}}(\mathrm{Pa})$ & $\varrho_{\mathrm{R}}\left(\mathrm{kg} / \mathrm{m}^{3}\right)$ & $v_{\mathrm{R}}(\mathrm{m} / \mathrm{s})$ & $p_{\mathrm{R}}(\mathrm{Pa})$ & $t_{\mathrm{f}}(\mathrm{s})$ \\
\hline 3.857 & 0.92 & 10.333 & 1.000 & 3.55 & 1.000 & 0.09 \\
\hline
\end{tabular}

Table 6

Settings for the Mach 3 shock test of Sec. 7.5. 

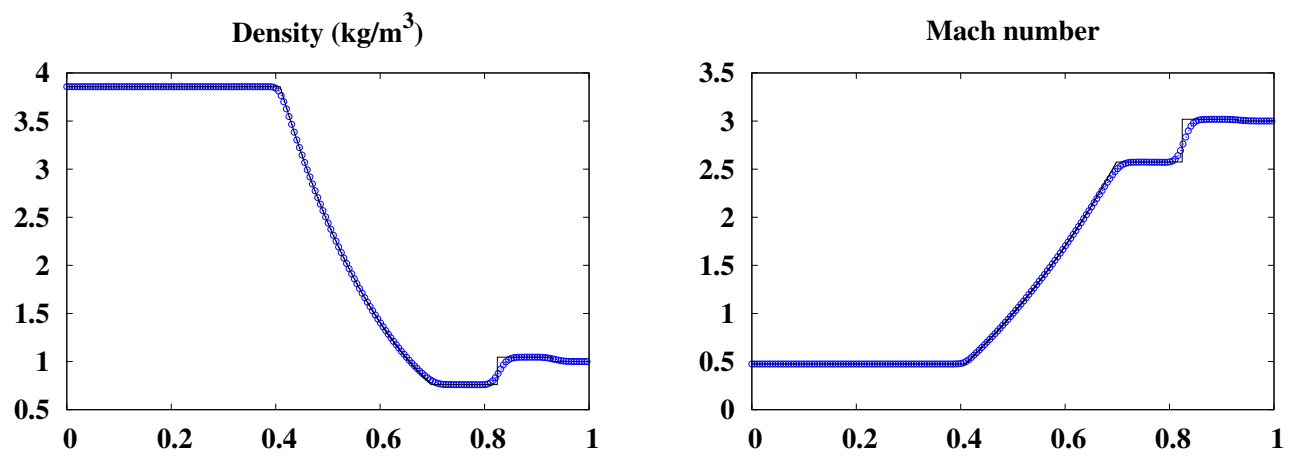

Fig. 15. Mach 3 shock: MIAU scheme.

\subsection{High Mach number shock tube}

This test, proposed in [38], allows to check the ability of the method to capture shocks at high Mach number. The initial data are listed in Table 7. The solution consists of two shocks and a contact discontinuity driven by a flow at a Mach number equal to 239. We take $N=800$ cells and $\mathrm{CFL}\left(v_{\mathrm{L}}\right)=0.5$.

\begin{tabular}{|c|c|c||c|c|c||c|}
\hline$\varrho_{\mathrm{L}}\left(\mathrm{kg} / \mathrm{m}^{3}\right)$ & $v_{\mathrm{L}}(\mathrm{m} / \mathrm{s})$ & $p_{\mathrm{L}}(\mathrm{Pa})$ & $\varrho_{\mathrm{R}}\left(\mathrm{kg} / \mathrm{m}^{3}\right)$ & $v_{\mathrm{R}}(\mathrm{m} / \mathrm{s})$ & $p_{\mathrm{R}}(\mathrm{Pa})$ & $t_{\mathrm{f}}(\mathrm{s})$ \\
\hline 10 & 2000 & 500 & 20 & 0 & 500 & $1.75 \times 10^{-4}$ \\
\hline
\end{tabular}

Table 7

Settings for the high Mach number shock tube test of Sec. 7.6.

The good results are shown in Fig. 16. The AUSM ${ }^{+}$and $\mathrm{AUSM}^{+}$-up schemes function also well (not shown). It is worth noticing that the convergence is lost if an interpolated face velocity is used instead of the $\mathrm{AUSM}^{+}$velocity in Eq. (31).

\section{Interaction of blast waves}

The interaction of blast waves considered by Woodward and Colella [42] has been simulated. This test case is very challenging since it features multiple strong shock waves. Such a configuration has also been selected for testing by Kwatra et al. [43] and Denner et al. [44].

The initial conditions at $t=0$ are

$$
\left(\varrho\left(\mathrm{kg} / \mathrm{m}^{3}\right), v(\mathrm{~m} / \mathrm{s}), p(\mathrm{~Pa})\right)= \begin{cases}\left(1,0,10^{3}\right), & 0 \leq x<0.1 \\ \left(1,0,10^{-2}\right), & 0.1 \leq x<0.9 \\ \left(1,0,10^{2}\right), & 0.9 \leq x \leq 1\end{cases}
$$



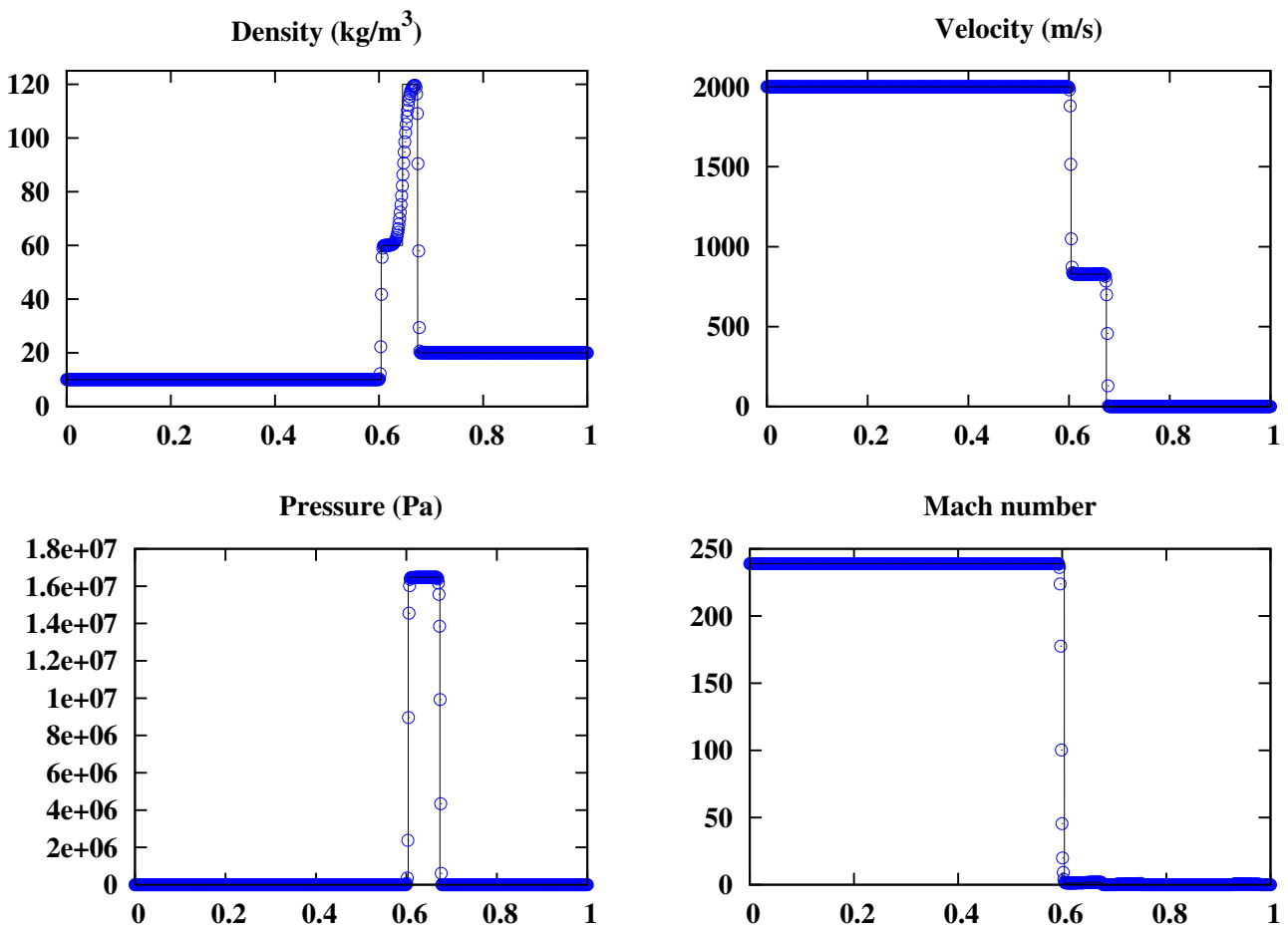

Fig. 16. High Mach number shock tube: MIAU scheme.

and solid wall boundary conditions are imposed at both sides of the domain. The solution shown at $t_{\mathrm{f}}=0.038 \mathrm{~s}$ in Fig. 17 has been calculated with $\operatorname{CFL}\left(v_{\mathrm{L}}\right)=0.5$ for three different uniform meshes of increasing size, from $N=800$ to $N=10000$, the latter being considered as our reference solution. According to Denner et al. [44], the results obtained by Woodward and Colella [42] with an adapted non-uniform mesh of 3096 cells can be considered as equivalent in resolution to a 24768 -cell uniform mesh. In the present calculations, it is visible that the solution obtained with the medium size mesh is already very close to our reference solution, whose quality is in line with those reported in the aforementioned studies.

\section{Tests of acoustic propagation in low Mach number flows}

In this section, we check the ability of the method to address the acoustic low Mach number limit of the Euler equations. We still use the Minmod slope limiter and secondorder backward differencing is used for time integration.

\subsection{One-dimensional acoustic pulse propagation in a low Mach number flow}

This test, proposed in [7] - see also [8] - allows verification of the dissipative and dispersive features of the method for acoustic waves propagating in low Mach number 

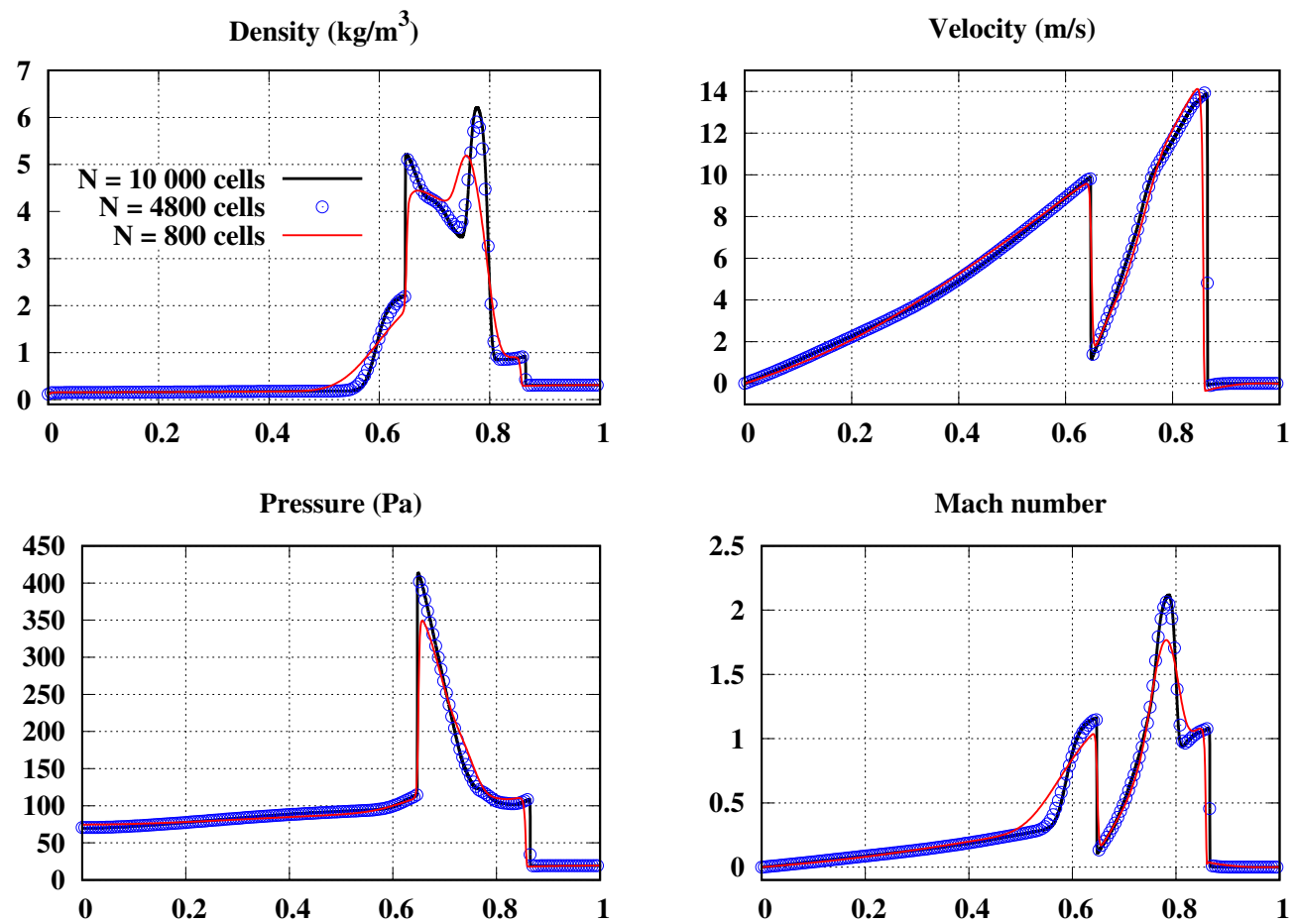

Fig. 17. Blast waves: MIAU scheme.

flows. The background flow is of constant density $\varrho_{0}=1.2046 \mathrm{~kg} / \mathrm{m}^{3}$, velocity $v_{0}=$ $3.0886 \times 10^{-2} \mathrm{~m} / \mathrm{s}$ and pressure $p_{0}=101300 \mathrm{~Pa}$. The corresponding Mach number is $10^{-4}$. A Gaussian pulse is generated at $t=0$ by

$$
\varrho^{0}=\varrho_{0}+(\delta \varrho)^{0}, \quad v^{0}=v_{0}+(\delta v)^{0}, \quad p^{0}=p_{0}+(\delta p)^{0},
$$

where

$$
\begin{aligned}
& (\delta p)^{0}=200 \exp \left[\frac{(x-0.2)^{2}}{2 \sigma^{2}}\right](\mathrm{Pa}), \sigma=2 \times 10^{-2} \mathrm{~m}, \\
& (\delta \varrho)^{0}=(\delta p)^{0} / c_{0}^{2}, \quad(\delta v)^{0}=(\delta p)^{0} /\left(\varrho_{0} c_{0}\right), \text { with } c_{0}=\sqrt{\gamma p_{0} / \varrho_{0}} .
\end{aligned}
$$

The computational domain is the interval $[0,5](\mathrm{m})$ divided into 2500 cells of equal length. $\operatorname{CFL}\left(v_{0}+c_{0}\right)=0.5$ and the solution is shown at $t_{\mathrm{f}}=11.07 \mathrm{~ms}$.

The very low dissipation by the MIAU scheme is shown in Fig. 18. The AUSM ${ }^{+}$and $\mathrm{AUSM}^{+}$-up schemes are dissipative, as shown in Figs. 19 and 20. For this test, the $\mathrm{AUSM}^{+}-$ up scheme does not succeed with the typical value 0.25 for the parameter $K_{p}$. This parameter had to be diminished until 0.1 .

An illustration of the $k$-iteration convergence in max-norm of the pressure correction and momentum correction is shown in Fig. 21 for the time-step 100, taken as an arbitrary example. The machine zero is obtained for the max-norm starting from iteration 5 for pressure correction, and iteration 4 for momentum correction. The choice to apply no more than 5 iteration per time-step is justified for the considered continuous solution. 


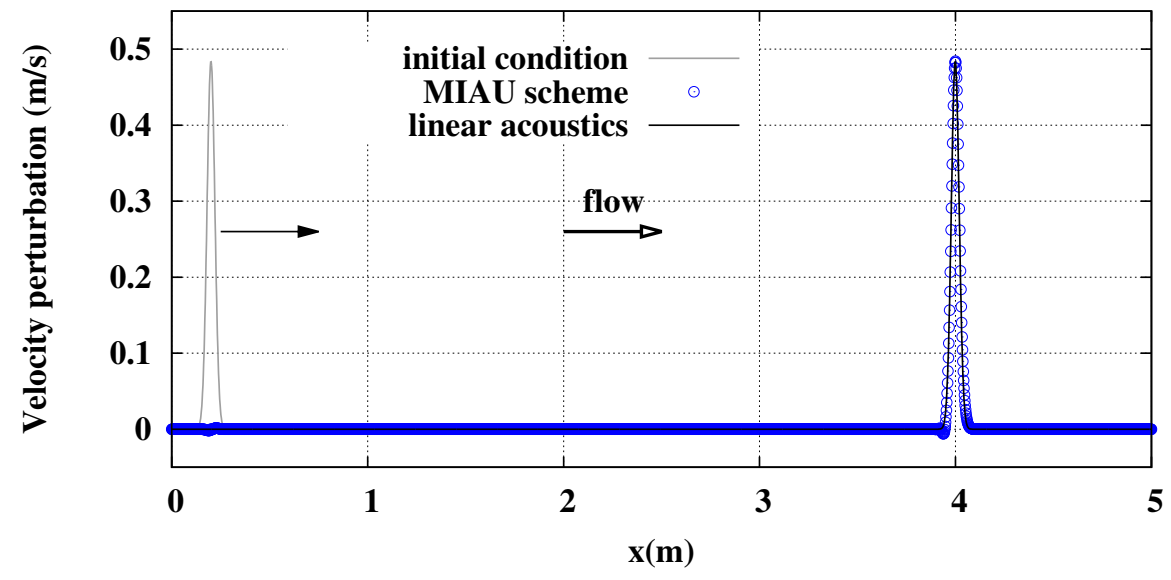

Fig. 18. One-dimensional acoustic pulse: MIAU scheme.

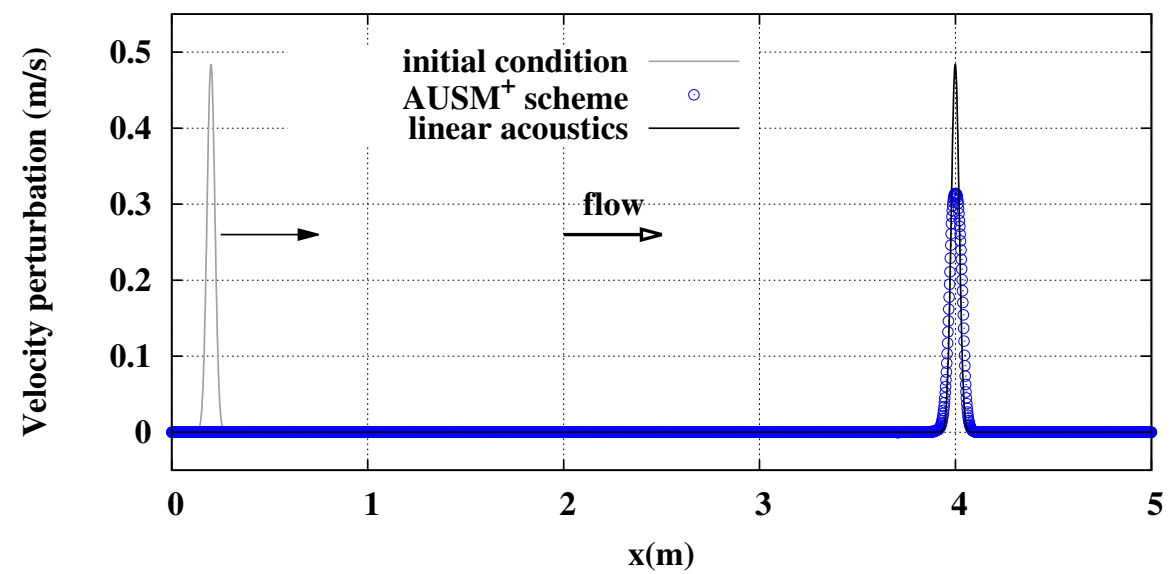

Fig. 19. One-dimensional acoustic pulse: $\mathrm{AUSM}^{+}$scheme.

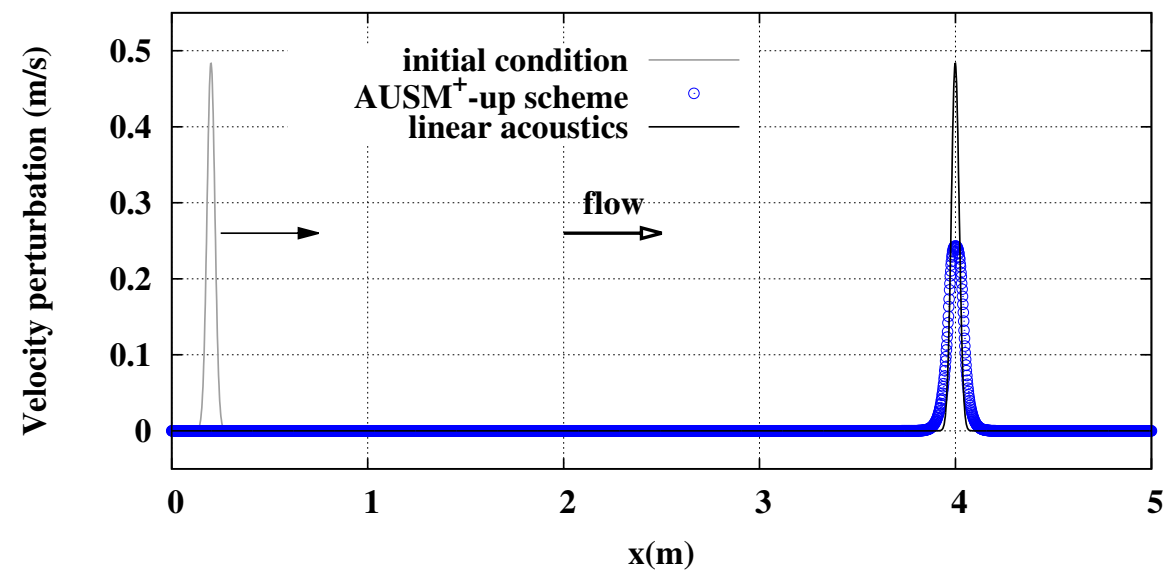

Fig. 20. One-dimensional acoustic pulse: $\mathrm{AUSM}^{+}$-up scheme with $K_{p}=0.1$, $f_{c}\left(M_{0}\right)=M_{0}\left(2-M_{0}\right)$ and $M_{\infty}=10^{-4}$.

From the several numerical tests we performed, this choice does appear sufficient for obtaining good quality solutions for all the tests considered in the present paper. 


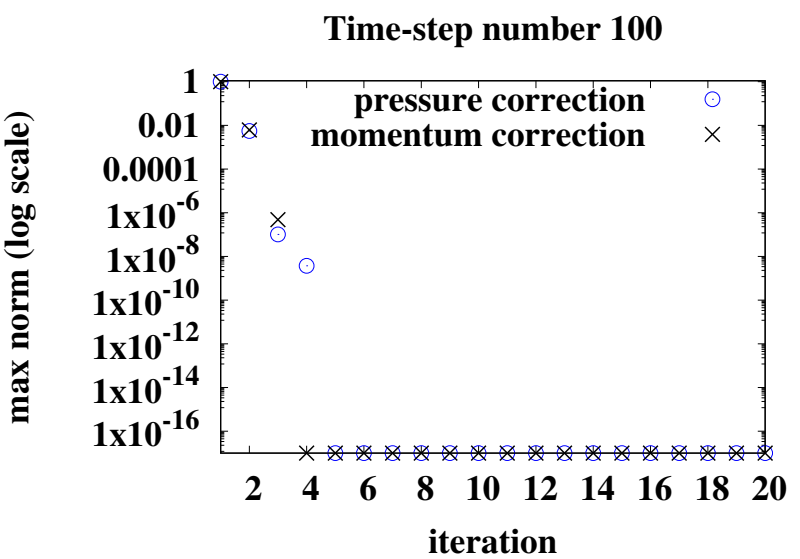

Fig. 21. One-dimensional acoustic pulse: MIAU scheme. $k$-iteration convergence in max-norm (logarithmic scale) at the time-step 100 with 20 iterations.

9.2 Two-dimensional acoustic pulse propagation in a low Mach number flow

This test, proposed in [45], allows to check the dissipative features of the method for multi-dimensional propagation of acoustic waves in low Mach number flows. With

$$
\varrho_{0}=1.2046 \mathrm{~kg} / \mathrm{m}^{3}, u_{0}=v_{0}=3.0886 \times 10^{-3} \mathrm{~m} / \mathrm{s}, p_{0}=101300 \mathrm{~Pa} \text {, }
$$

a two-dimensional Gaussian acoustic pulse is given at $t=0$ by

$$
\varrho^{0}=\varrho_{0}+(\delta \varrho)^{0}, u^{0}=u_{0}, v^{0}=v_{0}, p^{0}=p_{0}+(\delta p)^{0},
$$

where

$$
\begin{aligned}
& (\delta p)^{0}=A \exp \left\{-\alpha\left[\left(x^{\prime}\right)^{2}+\left(y^{\prime}\right)^{2}\right]\right\}(\mathrm{Pa}), \\
& \text { with } A=200, \alpha=1 /(0.05)^{2}, x^{\prime}=x-0.5, y^{\prime}=y-0.5, \\
& (\delta \varrho)^{0}=(\delta p)^{0} / c_{0}^{2} \text { and } c_{0}=\sqrt{\gamma p_{0} / \varrho_{0}} .
\end{aligned}
$$

For $t>0$, the speed of the acoustic wave generated by the initial pulse is the vector sum of the low Mach number mean flow velocity and the radially propagating sound speed. The exact solution of the initial value problem given by the Euler equations linearized around the uniform mean flow is (see Ref. [45]):

$$
\begin{aligned}
& \varrho\left(x^{\prime}, y^{\prime}, t\right)=\frac{A}{2 \alpha c_{0}^{2}} \int_{0}^{\infty} \exp \left(-\frac{\xi^{2}}{4 \alpha}\right) \cos \left(\xi c_{0} t\right) \mathrm{j}_{0}(\xi \eta) \xi \mathrm{d} \xi, \\
& u\left(x^{\prime}, y^{\prime}, t\right)=\frac{A\left(x^{\prime}-\mathrm{M}_{x} c_{0} t\right)}{2 \alpha \eta \rho_{0} c_{0}} \int_{0}^{\infty} \exp \left(-\frac{\xi^{2}}{4 \alpha}\right) \sin \left(\xi c_{0} t\right) \mathrm{j}_{1}(\xi \eta) \xi \mathrm{d} \xi, \\
& v\left(x^{\prime}, y^{\prime}, t\right)=\frac{A\left(y^{\prime}-\mathrm{M}_{y} c_{0} t\right)}{2 \alpha \eta \rho_{0} c_{0}} \int_{0}^{\infty} \exp \left(-\frac{\xi^{2}}{4 \alpha}\right) \sin \left(\xi c_{0} t\right) \mathrm{j}_{1}(\xi \eta) \xi \mathrm{d} \xi, \\
& p\left(x^{\prime}, y^{\prime}, t\right)=c_{0}^{2} \varrho\left(x^{\prime}, y^{\prime}, t\right),
\end{aligned}
$$


where

$$
\eta=\sqrt{\left(x^{\prime}-\mathrm{M}_{x} c_{0} t\right)^{2}+\left(y^{\prime}-\mathrm{M}_{y} c_{0} t\right)^{2}},
$$

and $\mathrm{j}_{0}$ and $\mathrm{j}_{1}$ are the first kind cylindrical Bessel functions of orders zero and one, respectively. Here, $\mathrm{M}_{x}=\mathrm{M}_{y}=v_{0} / c_{0}=9 \times 10^{-6}$. The computational domain is a square of side $[0,1](\mathrm{m})$, divided into $500 \times 500$ cells forming a regular Cartesian grid. The convective CFL number is $5 \times 10^{-5}$, and the corresponding acoustic CFL number is about 5 . The solution is shown at $t_{\mathrm{f}}=0.5$ and $1 \mathrm{~ms}$. The numerical method is the 2-D direct extension of the one detailed in section 3 , with an alternate direction procedure for solving the pentadiagonal system obtained from the energy equation.

\section{Pressure distribution (Pa)}

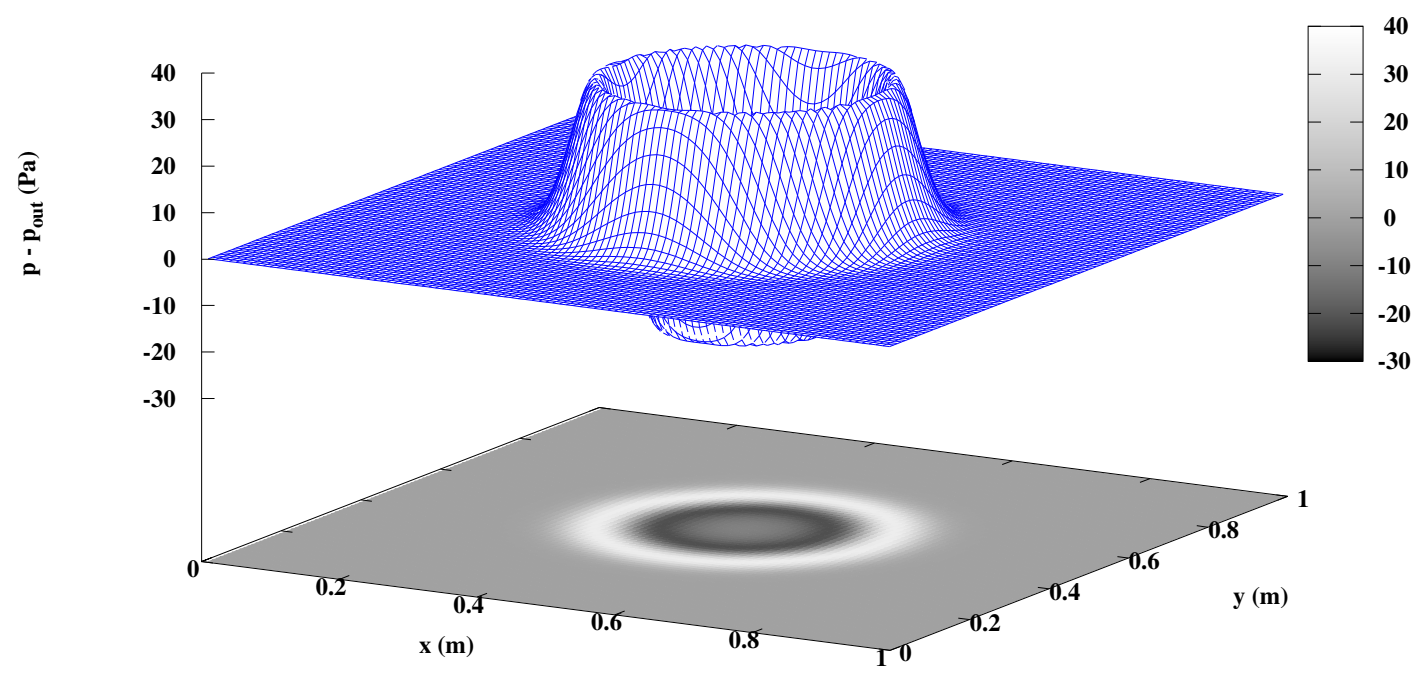

Fig. 22. Two-dimensional acoustic pulse test: MIAU scheme. Pressure distribution (Pa); $t_{f}=0.5 \mathrm{~ms}$.

The obtained pressure distributions are shown in Figs. $22\left(t_{f}=0.5 \mathrm{~ms}\right)$ and $23\left(t_{f}=\right.$ $1 \mathrm{~ms})$. The good results, obtained with the acoustic CFL number larger than unity, are shown in Figs. 24 and $26\left(t_{f}=0.5 \mathrm{~ms}\right)$, and 25 and $27\left(t_{f}=1 \mathrm{~ms}\right)$.

\section{Conclusion}

A pressure-correction algorithm for simulating fluid motion in a fully compressible framework is presented. It co-locates variables in space and incorporates the specifically devel- 


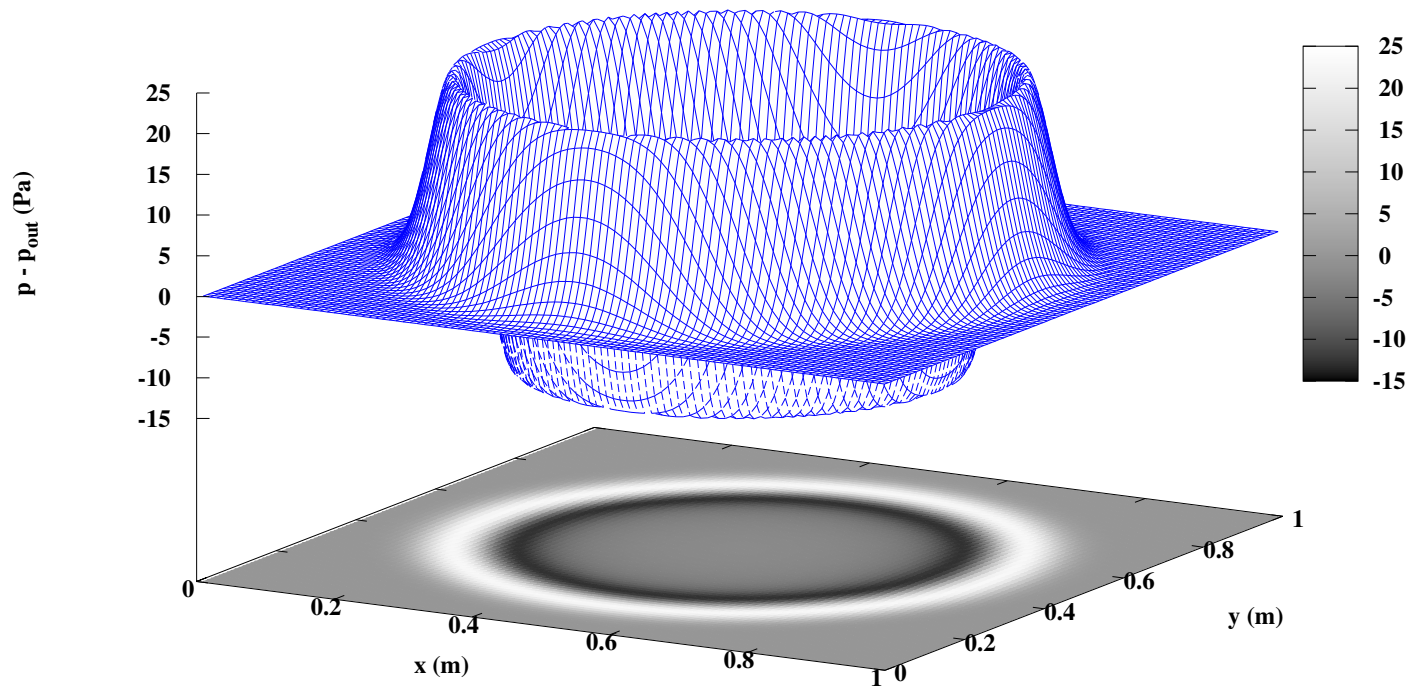

Fig. 23. Two-dimensional acoustic pulse test; MIAU scheme. Pressure distribution (Pa); $t_{f}=1 \mathrm{~ms}$.

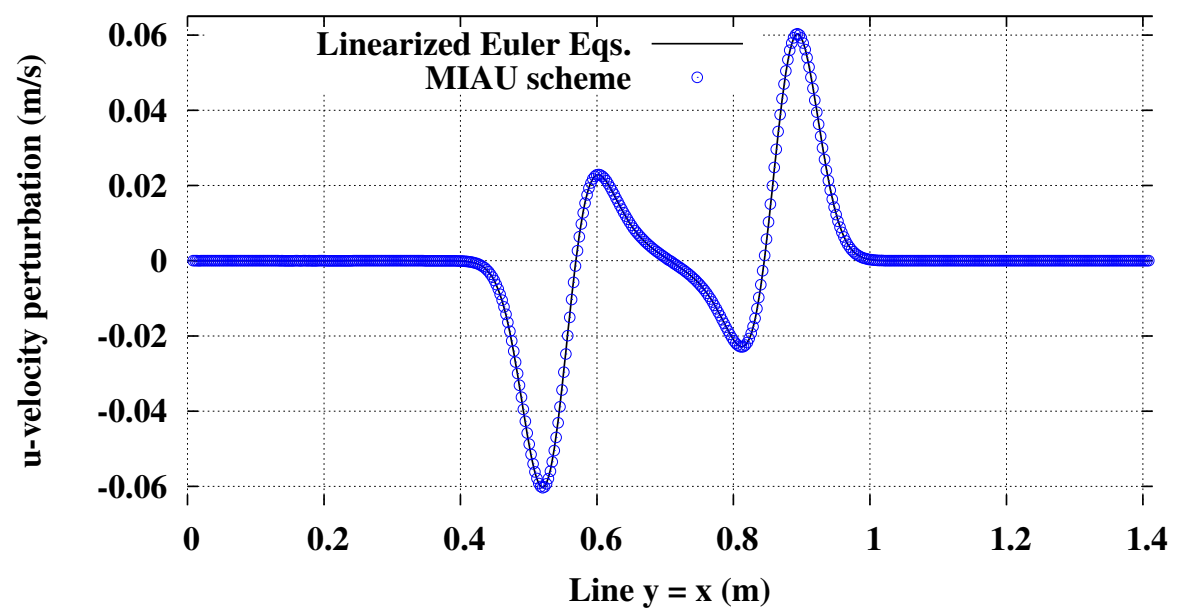

Fig. 24. Two-dimensional acoustic pulse test: MIAU scheme. $u$-velocity on $y=x$ (results for $v$-velocity are identical); $t_{f}=0.5 \mathrm{~ms}$.

oped MIAU flux scheme which combines a basic $\mathrm{AUSM}^{+}$flux splitting discretization, a face velocity derived from a time-consistent momentum interpolation and a face pressure derived from characteristic theory, but modified for obtaining the correct Mach number scaling in the low Mach limit. The different simulations of 1-D and 2-D steady or unsteady flows configurations demonstrate that the algorithm is able to provide good results over a very wide range of Mach number. The versatility of the proposed algorithm makes 


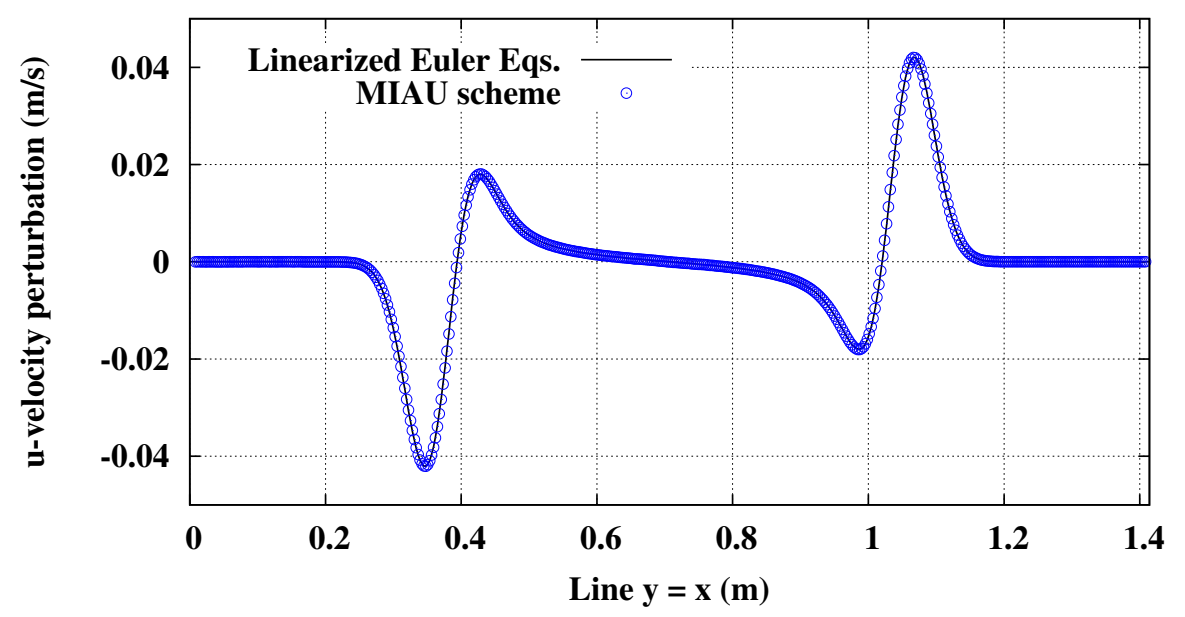

Fig. 25. Two-dimensional acoustic pulse test; MIAU scheme. $u$-velocity on $y=x$ (results for $v$-velocity are identical); $t_{f}=1 \mathrm{~ms}$.

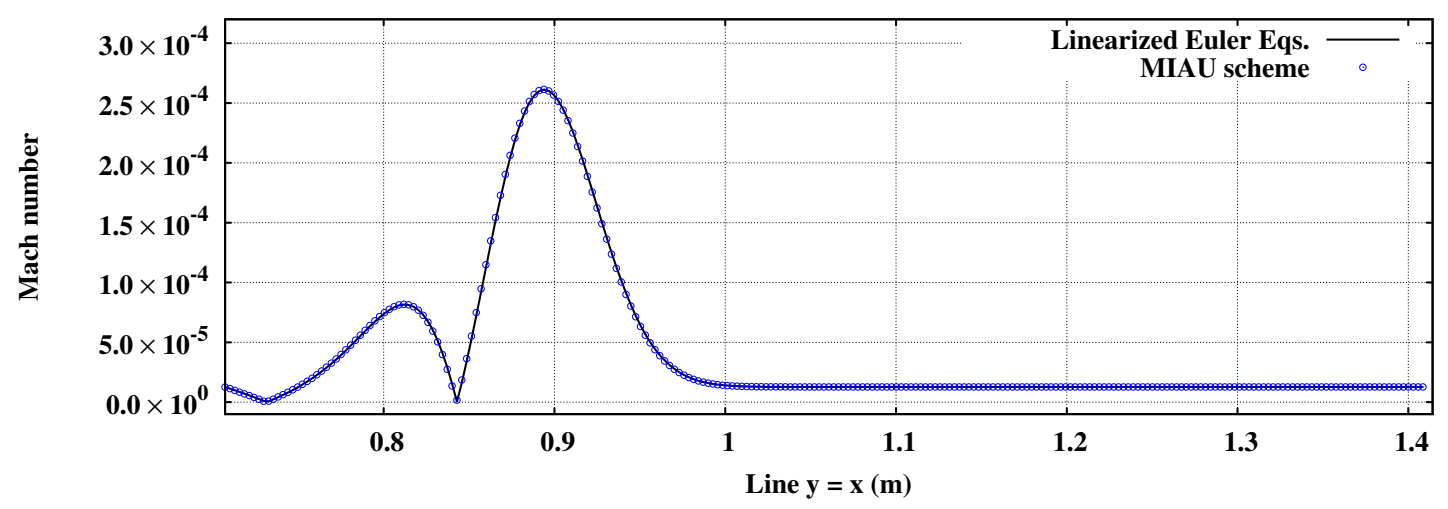

Fig. 26. Two-dimensional acoustic pulse test; MIAU scheme. Mach number $\sqrt{u^{2}+v^{2}} / c$ on $y=x$ (half of the computational domain, the solution being symmetric); $t_{f}=0.5 \mathrm{~ms}$.

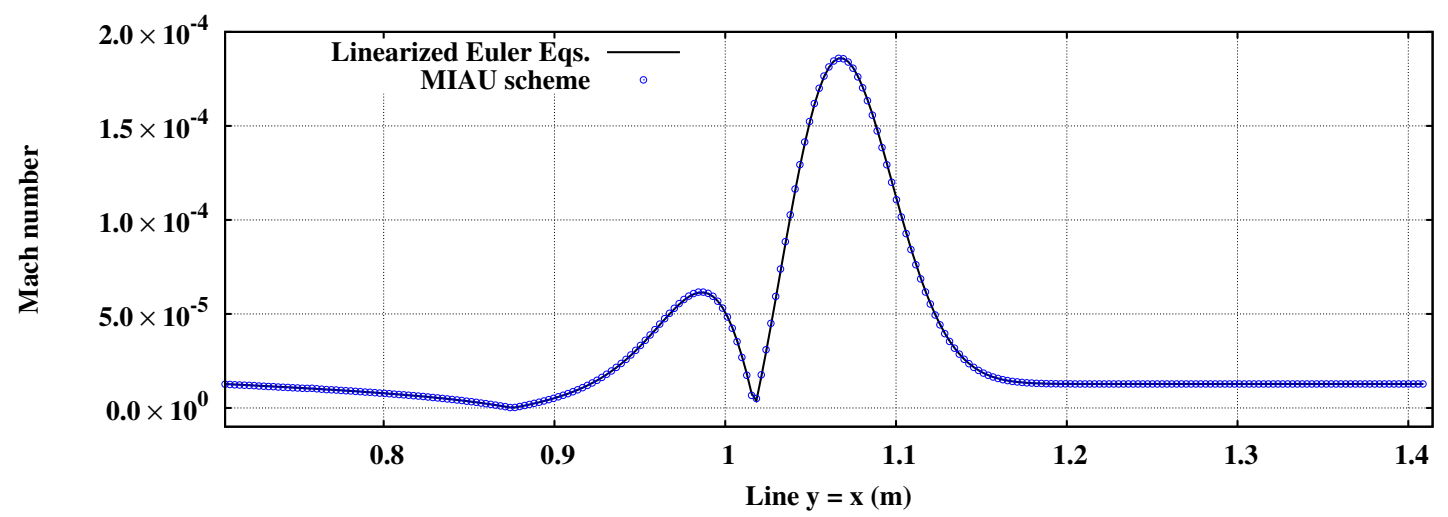

Fig. 27. Two-dimensional acoustic pulse test; MIAU scheme. Mach number $\sqrt{u^{2}+v^{2}} / c$ on $y=x$ (half of the computational domain, the solution being symmetric); $t_{f}=1 \mathrm{~ms}$.

it a valuable tool to investigate complex interactions encountered for instance in aeroacoustics. 


\section{A The $\mathrm{AUSM}^{+}$and $\mathrm{AUSM}^{+}$-up schemes}

\section{A.1 $\quad$ AUSM $^{+}-$up}

- Face velocity:

$$
v_{i+1 / 2}=c_{i+1 / 2} M_{i+1 / 2}-\frac{K_{p}}{\varrho_{i+1 / 2} c_{i+1 / 2} f_{c}\left(M_{0}\right)} \max \left\{1-\sigma \bar{M}^{2}, 0\right\}\left(p_{\mathrm{R}}-p_{\mathrm{L}}\right),
$$

with

$$
\begin{aligned}
& \varrho_{i+1 / 2}=\frac{1}{2}\left(\varrho_{\mathrm{L}}+\varrho_{\mathrm{R}}\right), c_{i+1 / 2}=\min \left\{\tilde{c}_{\mathrm{L}}, \tilde{c}_{\mathrm{R}}\right\}, \bar{M}^{2}=\frac{v_{\mathrm{L}}^{2}+v_{\mathrm{R}}^{2}}{2 c_{i+1 / 2}^{2}}, \\
\tilde{c}_{\mathrm{L}}= & \left(c_{\mathrm{L}}^{*}\right)^{2} / \max \left\{c_{\mathrm{L}}^{*}, v_{\mathrm{L}}\right\}, \tilde{c}_{\mathrm{R}}=\left(c_{\mathrm{R}}^{*}\right)^{2} / \max \left\{c_{\mathrm{R}}^{*},-v_{\mathrm{R}}\right\},\left(c_{\mathrm{L}, \mathrm{R}}^{*}\right)^{2}=\frac{2(\gamma-1)}{\gamma+1} H_{\mathrm{L}, \mathrm{R}}, \quad \text { (A.2b) } \\
& M_{i+1 / 2}=f_{M}^{+}\left(M_{\mathrm{L}}\right)+f_{M}^{-}\left(M_{\mathrm{R}}\right), f_{M}^{ \pm}(m)= \begin{cases}\frac{1}{2}(m \pm|m|) & ,|m| \geq 1, \\
\pm \frac{1}{4}(m \pm 1)^{2} \pm \frac{1}{8}\left(m^{2}-1\right)^{2} & ,|m|<1,\end{cases} \\
& M_{\mathrm{L}, \mathrm{R}}=v_{\mathrm{L}, \mathrm{R}} / c_{i+1 / 2}, \\
& f_{c}\left(M_{0}\right)=M_{0}\left(2-M_{0}\right), M_{0}^{2}=\min \left\{1, \max \left\{\bar{M}^{2}, \mathrm{M}_{\infty}^{2}\right\}\right\}, \\
& 0 \leq K_{p} \leq 1, \sigma \leq 1 ; \text { typically: } K_{p}=0.25, \sigma=1 .
\end{aligned}
$$

- Face pressure:

$$
\begin{aligned}
p_{i+1 / 2}=f_{p, \alpha}^{+}\left(M_{\mathrm{L}}\right) p_{\mathrm{L}} & +f_{p, \alpha}^{-}\left(M_{\mathrm{R}}\right) p_{\mathrm{R}} \\
& -2 K_{v} f_{c}\left(M_{0}\right) f_{p, \alpha}^{+}\left(M_{\mathrm{L}}\right) f_{p, \alpha}^{-}\left(M_{\mathrm{R}}\right) \varrho_{i+1 / 2} c_{i+1 / 2}\left(v_{\mathrm{R}}-v_{\mathrm{L}}\right),
\end{aligned}
$$

with

$$
\begin{aligned}
& f_{p, \alpha}^{ \pm}(m)= \begin{cases}\frac{1}{2}(1 \pm \operatorname{sign}(m)) & ,|m| \geq 1 \\
\frac{1}{4}(m \pm 1)^{2}(2 \mp m) \pm \alpha m\left(m^{2}-1\right)^{2} & ,|m|<1\end{cases} \\
& \alpha=\frac{3}{16}\left[-4+5\left(f_{c}\left(M_{0}\right)\right)^{2}\right] \\
& 0 \leq K_{v} \leq 1 ; \text { typically: } K_{v}=0.75
\end{aligned}
$$

- Convected quantities: upwinded.

\section{A.2 $\mathrm{AUSM}^{+}$}

Following Liou [19], the $\mathrm{AUSM}^{+}$scheme is a special case of the $\mathrm{AUSM}^{+}$-up scheme, by taking $K_{p}=0$ in Eq. (A.1), $K_{v}=0$ and $\alpha=3 / 16$ in Eq. (A.3), with the interface speed of sound defined by Eqs. (19) and (20). 
The interface speed of sound for the $\mathrm{AUSM}^{+}$-up scheme [19] (Eqs. (A.2)) is different to the interface speed of sound initially proposed by Liou in [18] for the $\mathrm{AUSM}^{+}$scheme (Eqs. (19)-(20)). The modification proposed in [19] allows to address the problem of entropy-violating jumps, and essentially consists in taking into account the flow direction. Since the information of the flow direction is incorporated in the face velocity of the MIAU scheme by the upwinding of the momentum equation, the original definition of the interface speed of sound proposed in [18] was retained in Eq. (20).

\section{References}

[1] R. J. LeVeque. Finite volume methods for hyperbolic problems. Cambridge University Press, 2002, ISBN 13-978-0-521-81087-6.

[2] E. F. Toro. Riemann solvers and numerical methods for fluid dynamics. Springer, third edition, 2009, ISBN 978-3-540-49834-6.

[3] P. G. Tucker. Advanced computational fluid and aerodynamics. Cambridge University Press, 2016, ISBN 978-1-107-42883-6.

[4] P. Wesseling. Principles of computational fluid dynamics. Springer, 2009, ISBN 978-3-54067853-3.

[5] S. Acharya, B. R. Baliga, K. Karki, J. Y. Murthy, and C. Prakash. Pressure-based finitevolume methods in computational fluid dynamics. J. Heat Transfer, 129:407-424, 2007.

[6] R. Klein. Semi-implicit extension of a Godunov-type scheme based on low Mach number asymptotics I:One-dimensional flow. J. Comput. Phys., 121:213-237, 1995.

[7] Y. Moguen, T. Kousksou, P. Bruel, J. Vierendeels, and E. Dick. Pressure-velocity coupling allowing acoustic calculation in low Mach number flow. J. Comput. Phys., 231:5522-5541, 2012.

[8] Y. Moguen, S. Delmas, V. Perrier, P. Bruel, and E. Dick. Godunov-type schemes with an inertia term for unsteady full Mach number range flow calculations. J. Comput. Phys., 281:556-590, 2015.

[9] C. M. Rhie and W. L. Chow. Numerical study of the turbulent flow past an airfoil with trailing edge separation. AIAA J., 21:1525-1532, 1983.

[10] S. Majumdar. Role of underrelaxation in momentum interpolation for calculation of flow with nonstaggered grids. Numer. Heat Transfer A, 13:125-132, 1988.

[11] T. F. Miller and F. W. Schmidt. Use of a pressure-weighted interpolation method for the solution of the incompressible Navier-Stokes equations on a nonstaggered grid system. Numer. Heat Transfer A, 14:213-233, 1988.

[12] F. S. Lien and M. A. Leschziner. A general non-orthogonal collocated finite volume algorithm for turbulent flow at all speeds incorporating second-moment turbulence-transport closure, Part 1: Computational implementation. Comput. Methods Appl. Mech. Eng., 114:123-148, 1994. 
[13] A. Pascau. Cell face velocity alternatives in a structured colocated grid for the unsteady Navier-Stokes equations. Int. J. Numer. Methods Fluids, 65:812-833, 2011.

[14] K. C. Ong and A. Chan. A pressure-based Mach-uniform method for viscous fluid flows. Int. J. Comput. Fluid Dyn., 30:516-530, 2016.

[15] C.-N. Xiao, F. Denner, and B. G. M. van Wachem. Fully-coupled pressure-based finitevolume framework for the simulation of fluid flows at all speeds in complex geometries. $J$. Comput. Phys., 346:91-130, 2017.

[16] Y. Moguen, E. Dick, J. Vierendeels, and P. Bruel. Pressure-velocity coupling for unsteady low Mach number flow simulations: An improvement of the AUSM+-up scheme. J. Comput. Appl. Math., 246:136-143, 2013.

[17] Y. Moguen, P. Bruel, and E. Dick. Solving low Mach number Riemann problems by a momentum interpolation method. J. Comput. Phys., 298:741-746, 2015.

[18] M.-S. Liou. A sequel to AUSM: AUSM+. J. Comput. Phys., 129:364-382, 1996.

[19] M.-S. Liou. A sequel to AUSM, part II: AUSM+ -up for all speeds. J. Comput. Phys., 214:137-170, 2006.

[20] T. Yabe and P.-Y. Wang. Unified numerical procedure for compressible and incompressible fluid. J. Phys. Soc. Jpn., 60:2105-2108, 1991.

[21] G.-C. Zha and E. Bilgen. Numerical solution of Euler equations by using a new flux vector splitting scheme. Int. J. Numer. Methods Fluids, 17:115-155, 1993.

[22] E. F. Toro and M. E. Vázquez-Cendón. Flux splitting schemes for the Euler equations. Comput. Fluids, 70:1-12, 2012.

[23] R. G. Rehm and H. R. Baum. The equations of motion for thermally driven, boyuant flows. N.B.S.J. Res., 83:293-308, 1978.

[24] A. Majda and J. Sethian. The derivation and numerical solution of the equations for zero Mach number combustion. Combust. Sci. Technol., 42:185-205, 1985.

[25] A. Meister. Asymptotic single and multiple scale expansions in the low Mach number limit. SIAM J. Appl. Math., 60:256-271, 1999.

[26] J. R. Edwards and M.-S. Liou. Low-diffusion flux-splitting methods for flows at all speeds. AIAA J., 36:1610-1617, 1998.

[27] E. Shima and K. Kitamura. Parameter-free simple low-dissipation AUSM-family scheme for all speeds. AIAA J., 49:1693-1709, 2011.

[28] K. Kitamura and E. Shima. Towards shock-stable and accurate hypersonic heating computations: a new pressure flux for AUSM-family schemes. J. Comput. Phys., 245:6283, 2013.

[29] F. Rieper. A low-Mach number fix for Roe's approximate Riemann solver. J. Comput. Phys., 230:5263-5287, 2011.

[30] X.-S. Li and C.-W. Gu. Mechanism of Roe-type schemes for all-speed flows and its application. Comput. fluids, 86:56-70, 2013. 
[31] H. Guillard and C. Viozat. On the behaviour of upwind schemes in the low Mach number limit. Comput. Fluids, 28:63-86, 1999.

[32] X.-S. Li and C.-W. Gu. The momentum interpolation method based on the time-marching algorithm for all-speed flows. J. Comput. Phys., 229:7806-7818, 2010.

[33] W. Z. Shen, J. A. Michelsen, and J. N. Sørensen. Improved Rhie-Chow interpolation for unsteady flow computations. AIAA J., 39:2406-2409, 2001.

[34] F. Denner and B. G. M. van Wachem. Fully coupled balanced-force VOF framework for arbitrary meshes with least-squares curvature evaluation from volume fractions. Numer. Heat Transfer B-Fund., 65:218-255, 2014.

[35] P. Bartholomew, F. Denner, M. H. Abdol-Azis, A. Marquis, and B. G. M. van Wachem. Unified formulation of the momentum-weighted interpolation for collocated variable arrangements. J. Comput. Phys., 375:177-208, 2018.

[36] F. Denner. Fully coupled pressure-based algorithm for compressible flows: Linearisation and iterative solution strategies. Comput. Fluids, 175:53-65, 2018.

[37] D. R. van der Heul, C. Vuik, and P. Wesseling. A conservative pressure-correction method for flow at all speeds. Comput. Fluids, 32:1113-1132, 2003.

[38] F. Xiao. Unified formulation for compressible and incompressible flows by using multiintegrated moments I: One-dimensional inviscid compressible flow. J. Comput. Phys., 195:629-654, 2004.

[39] J. W. Banks, T. Aslam, and W. J. Rider. On sub-linear convergence for linearly degenerate waves in capturing schemes. J. Comput. Phys., 227:6985-7002, 2008.

[40] H. Guillard and A. Murrone. On the behavior of upwind schemes in the low Mach number limit: II. Godunov type schemes. Comput. Fluids, 33:655-675, 2004.

[41] M. Arora and P. Roe. A well-behaved TVD limiter for high resolution calculations of unsteady flow. J. Comput. Phys., 132:3-11, 1997.

[42] P. Woodward and P. Colella. The numerical simulation of two-dimensional fluid flow with strong shocks. J. Comput. Phys., 54:115-173, 1984.

[43] N. Kwatra, J. Su, J. T. Grétarsson, and R. Fedwik. A method for avoiding the acoustic time step restriction in compressible flow. J. Comput. Phys., 228:4146-4161, 2009.

[44] F. Denner, C. N. Xiao, and B. G. M. van Wachem. Pressure-based algorithm for compressible interfacial flows with acoustically-conservative interface discretisation. J. Comput. Phys., 367:192-234, 2018.

[45] C. K. W. Tam and J. C. Webb. Dispersion-relation-preserving finite difference schemes for computational acoustics. J. Comput. Phys., 107:262-281, 1993. 